\title{
Electronic Structure of the fcc Transition Metals Ir, Rh, Pt, and Pd
}

\author{
Andersen, O. Krogh
}

Published in:

Physical Review B

Link to article, DOI:

10.1103/PhysRevB.2.883

Publication date:

1970

Document Version

Publisher's PDF, also known as Version of record

Link back to DTU Orbit

Citation (APA):

Andersen, O. K. (1970). Electronic Structure of the fcc Transition Metals Ir, Rh, Pt, and Pd. Physical Review B, 2(4), 883-906. https://doi.org/10.1103/PhysRevB.2.883

\section{General rights}

Copyright and moral rights for the publications made accessible in the public portal are retained by the authors and/or other copyright owners and it is a condition of accessing publications that users recognise and abide by the legal requirements associated with these rights.

- Users may download and print one copy of any publication from the public portal for the purpose of private study or research.

- You may not further distribute the material or use it for any profit-making activity or commercial gain

- You may freely distribute the URL identifying the publication in the public portal

If you believe that this document breaches copyright please contact us providing details, and we will remove access to the work immediately and investigate your claim 
tance. What we used here instead - a Fermi surface having flat faces connected by simple types of curved surfaces - did make it possible to carry out a relatively simple computation of the Seitz magnetoresistance coefficients in a highly distorted, nonellipsoidal model. In all probability, no other kind of nonellipsoidal model would have led to a simpler calculation.
However, it will not be as easy to use this kind of model as an approximation to a variety of real Fermi surfaces as it was in the case of the Hall coefficient. But in view of the enormous complexity of most magnetoresistance calculations, we hope that this single example has provided some useful insight into the general behavior of weakfield magnetoresistance.

\footnotetext{
${ }^{*}$ Work supported in part by ONR Contract No. PO-90163.

${ }^{1}$ R. S. Allgaier, Phys. Rev. 165, 775 (1968).

${ }^{2}$ R. S. Allgaier, Phys. Rev. (to be published).

${ }^{3}$ See Ref. 1, note added in manuscript.

${ }^{4} \mathrm{H}$. Miyazawa, in Proceedings of the International Conference on the Physics of Semiconductors, Exeter (The Instiiute of Physics and the Physical Society, London, 1962), p. 636.

${ }^{5}$ A. C. Beer, Galvanomagnetic Effects in Semiconduc-
}

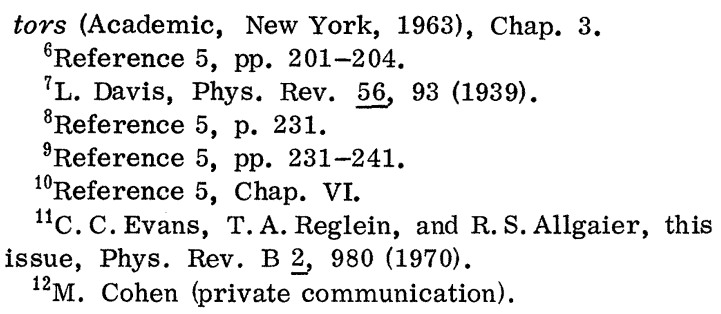

\author{
Electronic Structure of the fcc Transition Metals Ir, Rh, Pt, and $\mathrm{Pd}^{\dagger}$ \\ O. Krogh Andersen* \\ Laboratory for Electrophysics, Technical University, Lyngby, Denmark \\ and \\ Physics Department, University of Pennsylvania, Philadelphia, Pennsylvania 19104 \\ (Received 11 December 1969)
}

\begin{abstract}
We give a complete description of a relativistic augmented-plane-wave calculation of the band structures of the paramagnetic fec transition metals Ir, Rh, Pt, and Pd. The width and position of the $d$ band decrease in the sequence Ir, $\mathrm{Pt}, \mathrm{Rh}, \mathrm{Pd}$; and $N\left(E_{F}\right)=13.8,23.2,18.7$, and 32.7 (states/atom)/Ry, respectively. Spin-orbit coupling is important for all four metals and the coupling parameter varies by $30 \%$ over the $d$ bandwidth. Detailed comparisons with de Haas-van Alphen Fermi-surface dimensions have previously been presented and the agreement was very good. Comparison with measured electronic specific-heat coefficients $\gamma$ and cyclotron masses indicate that the average mass enhancements are $1.37,1.44,1.63$, and 1.66 for Ir, Rh, Pt, and Pd, respectively; and that for both Pt and Pd the partial enhancements on the closed electron surface and on the open hole surface are 1.51 and 1.68 , respectively. Saddle points in the fifth band are important for the large peak in $N(E)$ near the top of the $d$ band. The experimental $\gamma(x)$ for $\mathrm{Rh}_{x} \mathrm{Pd}_{1-x}$ is not uniformly enhanced over the calculated rigid-band $\gamma(x)$. The experimentally observed field and temperature dependences of the magnetic susceptibility for Pd are consistent with the calculated $N(E)$.
\end{abstract}

\section{INTRODUCTION}

In recent years there has been great interest in explaining the increasing tendency towards ferromagnetism and the simultaneous disappearance of superconductivity through the sequence of fcc $4 d$ and $5 d$ transition metals: $\mathrm{Ir}, \mathrm{Rh}, \mathrm{Pt}$, and $\mathrm{Pd} .^{1}$
Particular attention has been paid to the role of spin fluctuations, ${ }^{2}$ produced by the strong exchange interactions among the $d$ electrons. A starting point for the quantitative explanation of such effects is an accurate knowledge of the parameters of the one-electron theory for these metals, ${ }^{3,4}$ and we have therefore calculated surfaces 
of constant energy by the a priori relativistic augmented-plane-wave (RAPW) method $^{5}$ and thereby obtained accurate theoretical Fermi surfaces, cyclotron masses, and state densities at the Fermi level. In order to evaluate the extent to which the effective masses of the conduction electrons are enhanced by spin fluctuations, we have compared our theoretical values with de Haas-van Alphen (dHvA) and specific-heat measurements and found average enhancements, including the contribution from electron-phonon interactions, of 1.37, 1.44, 1. 63, and 1.66 for $\mathrm{Ir}, \mathrm{Rh}, \mathrm{Pt}$, and $\mathrm{Pd}$, respectively. ${ }^{6}$ Spin-fluctuation theories, ${ }^{2}$ which have so far only dealt crudely with wave-vector dependencies from band structure and exchange, have predicted that for $\mathrm{Pd}$ the spin fluctuations should contribute to the enhancement by a factor of at least 2 . This discrepancy tends to confirm that an understanding of the topology of the Fermi surfaces and of the distribution of band velocities thereon will be needed for a quantitative description of the manybody effects.

In the present paper, we give a detailed account of the previously published RAPW calculations ${ }^{6}$ and report further results. The computational details may be found in Sec. II and the energy eigenvalues at the points of high symmetry are given in Sec. III A. The shapes of the energy bands are very similar for all four metals and the differences may essentially be described in terms of positions and widths of $d$ bands ${ }^{7,8}$ and spin-orbit coupling strengths. The main part of Sec. III A is devoted to the relativistic effects; in particular, the RAPW spin-orbit splittings are compared with results derived from a tight-binding $d$ band perturbed by spin-orbit interaction. In doing so, we make use of a spin-orbit parameter, which is found from the relativistic equivalents of the logarithmic derivative, as described in the Appendixes. Here we also point out an inaccuracy in the usual interpretation of the RAPW matrix element. In Sec. III B we give the results for the Fermi surfaces, velocities, and partial statedensities. The high density of states for these metals is principally due to one particular flat band, the fifth band, and, since the topology of the corresponding Fermi-surface sheets of $\mathrm{Rh}$ and Ir ( 9 valence electrons) is very different from those of $\mathrm{Pd}$ and $\mathrm{Pt}$ (10 valence electrons), van Hove singularities must exist in the density of states between the Fermi levels corresponding to 9 and 10 electrons. We have traced constant-energy contours for the $\mathrm{Rh}$ and $\mathrm{Pd}$ bands near these saddle points in $E(\overrightarrow{\mathrm{k}})$ and have obtained the detailed density-of-states curves in the rigid-band approximation for $\mathrm{RhPd}$ and dilute PdAg alloys. This is reported in Sec. III C, and we point out that other irreducible zones than the conventional $\frac{1}{48}$ Brillouin zone exist, and may be more appropriate for $d$ bands; also the connection with the Fermi-surface sheet of the fifth band of ferromagnetic $\mathrm{Ni}$ is mentioned. In Sec. IV A we compare our total density of states with measurements of the electronic specificheat coefficient for the pure metals and, using the rigid-band approximation, for $\mathrm{RhPd}, \mathrm{PdAg}$, and $\mathrm{RhNi}$ alloys, and we derive the enhancement factors. We also consider the susceptibility data for $\mathrm{Pd}$ and $\mathrm{Pt}$, and for the former metal comparison with the field and temperature dependence of the measured susceptibility is made. Since the appearance of our preliminary publication, ${ }^{6} \mathrm{dHv} A$ data on $\mathrm{Ir}^{9}$ and more accurate $\mathrm{Pd}$ data ${ }^{10}$ have become available, and in Sec. IV B we give a measure of the agreement with our theoretical Fermisurface dimensions and deduce partial mass enhancements for the various Fermi-surface sheets for all four metals. In the discussion of Sec. V we try to give some justification of the rationale underlying the present a priori band-structure calculation: We believe that the Fermi surface itself is very little affected by the interactions with phonons and spin fluctuations. If we are therefore able to construct a physically reasonable oneelectron crystal potential, which yields a theoretical Fermi surface in full agreement with the experimental Fermi surface, the theoretical bands, masses, and state densities represent the "bare" or noninteracting values. It should be noted that the requirement of agreement with the experimental Fermi surface is by no means trivial, since it is well known ${ }^{11}$ that slightly different, physically reasonable, potentials for the same transition metal can yield vastly different Fermi surfaces and energy scales. In this Sec. $\mathrm{V}$ we shall further draw attention to the relativistic terms and for $\mathrm{Pd}$ and Pt try to evaluate their effect on the state densities.

\section{DETAILS OF CALCULATIONS}

The RAPW calculations were essentially performed by the methods described by Loucks. ${ }^{5}$ Muffin-tin potentials for $\mathrm{Rh}^{45}, \mathrm{Pd}^{46}, \mathrm{Ir}^{77}$, and $\mathrm{Pt}^{78}$ were constructed ${ }^{12,5}$ from self-consistent Dirac-Slater charge densities, ${ }^{13}$ calculated for the neutral atoms, ${ }^{14}$ and those configurations giving the minimum average energy of the atoms were chosen also for the solids; they may be found in Table I. Slater's $\rho^{1 / 3}$ free-electron approximation for exchange was used in its full strength, as preliminary bands based on atomic calculations and potential constructions with both full and reduced exchange ${ }^{15}$ revealed that the former yielded better agreement with experiments. No attempt was made to form self-consistent crystal potentials, 
TABLE I. Input data and results.

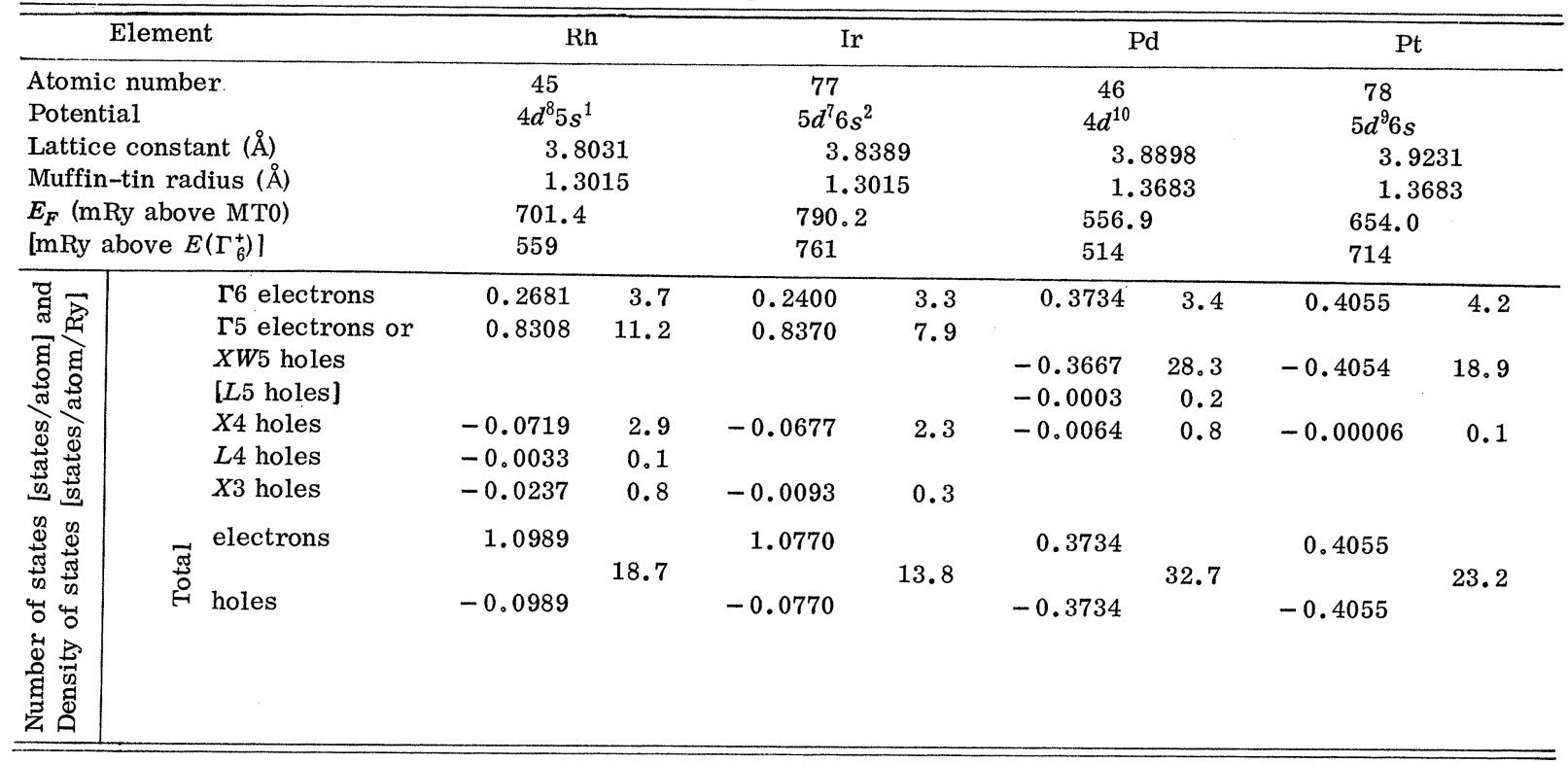

but their accuracy was rather judged by the extent of the agreement between the calculated and measured Fermi-surface dimensions and velocities.

All four crystals have the fcc structure with almost equal lattice constants (Table I). Nearly all the calculated Bloch vectors were found in the conventional irreducible zone, and with a few exceptions the number of basis functions in the RAPW expansion was 88 , i. e., 44 for each spin component. With this set of 44 reciprocal-lattice points the first excluded point is nearest to the symmetry point $-X$, the distance being $10^{1 / 2} \times|\Gamma X|$, which is about seven times the inverse of the muffin-tin radii applied. The basis was not symmetrized ${ }^{16}$ since our main interest was the constant-energy surfaces near the Fermi surface rather than energy eigenvalues and wave functions at points of high symmetry. The eigenvalues, except those in the high-energy region well above the top of the $d$ band, were converged to within $3 \mathrm{mRy}$ relative to the potential zero MT0 between muffin-tin spheres (MTS). The convergence of energy separations within the same or neighboring bands was far better, however, and a preliminary Pd calculation employing only 36 reciprocal-lattice vectors yielded the same Fermi surface, an $0.8 \mathrm{mRy}$ higher Fermi energy relative to MT0 and a $3 \%$ lower density of states at the Fermi level, than was obtained with the 44 reciprocal-lattice vectors.

Energies at the symmetry points and along some symmetry lines were calculated from the Dirac RAPW matrix in the formulation given by Eq. (A1). The summation over angular momenta is rapidly convergent for the $\beta$ term, because when $l$ is larger than the value 2 corresponding to the $d$-wave resonance, the centrifugal term of the radial equation [Eq. (A4)] effectively dominates the spin-orbit term, and the solutions corresponding to $j=l \pm \frac{1}{2}$ are nearly equal. It was found that for $l>2$ all $\beta$ 's were more than two orders of magnitude smaller than $\beta_{1}$; nevertheless the summation was not truncated until after the $l=4$ term (Fig. 16). Conver- gence of the $\alpha$ summation is, however, determined by the spherical Bessel functions and therefore depends on their argument, i.e., $\max |\overrightarrow{\mathrm{g}}+\overrightarrow{\mathrm{k}}|$. With the above 44 basis it was appropriate to truncate after the $l=8$ term. It might be mentioned, however, that faster convergence of the $\alpha$ term is obtained, for instance, with the nonsymmetrical form given by Loucks. ${ }^{5}$

The secular matrix was set up and the determinant $D_{\mathrm{k}}(E)$ evaluated by computer routines derived from those given by Loucks. The energies were found to an accuracy better than $0.3 \mathrm{mRy}$ by solving $D_{\overrightarrow{\mathrm{k}}}(E)=0$ by an iterative numerical procedure. Using a nonsymmetrized basis, the symmetry labeling of the various energy levels was partly determined by comparison with the $\mathrm{Cu}$ bands of Bur$\operatorname{dick}^{17}$ and with the tight-binding calculation including spin-orbit coupling on Pt by Friedel et al. ${ }^{18}$ Moreover, removal of the spin-orbit coupling using the approximate method of Mattheiss ${ }^{19}$ (see the Appendixes) identified the spin-orbit splittings.

For each metal, three or more constant-energy surfaces were traced out in the vicinity of the Fermi surface; they were separated by 1. 5-10 mRy depending on the corresponding density of states. The Fermi level $E_{F}$ was determined by 
setting the difference in volumes between electron and hole Fermi-surface sheets to one state per atom in $\mathrm{Rh}$ and $\mathrm{Ir}$ and to zero in $\mathrm{Pd}$ and $\mathrm{Pt}$ (see Sec. III B). The density of states $N\left(E_{F}\right)$ was obtained from the variation with energy of the volumes $V$ of constant-energy surfaces. As each constant-energy surface was calculated at about 150 points in the $\frac{1}{48}$ Brillouin zone, all by the firstprinciples RAPW method, and as the energy increments were small, the above procedure gives very accurate values of $N\left(E_{F}\right)$ for the assumed potential. Also the group velocities $\overrightarrow{\mathrm{v}}(\overrightarrow{\mathrm{k}})$ over the whole Fermi surface may be extracted from our results. However, we only explicitly calculated velocities in a few cases and rather concentrated on the cyclotron masses $m_{\hat{n}}$ and extremal areas $A_{\hat{n}}$. The constant-energy surfaces were traced by solving $D_{E}(\overrightarrow{\mathrm{k}})=0$ with $\overrightarrow{\mathrm{k}}$ expressed in suitable coordinates, spherical or cylindrical, depending on the surface under consideration. Two of the coordinates (e.g., $\varphi$ and $\theta$ ) were varied over a mesh compatible with the boundaries of the irreducible zone, whereas the zero of the determinant was found from variation of the third coordinate (e.g., $r$ ). In order to speed up the iterative procedure, extrapolation from previously calculated points of the constantenergy contour [e.g., $\left.r_{E}(\theta)\right]$ and from the values of the determinant around it was employed. The radii $r_{E}$ or $R_{E}$ were found to a minimum accuracy of $10^{-4}$ a.u. In order to compute accurate values for areas between adjacent constant-energy contours and volumes between adjacent constantenergy surfaces by numerical integration over as few points as possible, it was important to use the $(\varphi, \theta)$ mesh for all energies. Careful evaluation of the numerical accuracy ensured that all volumes, areas, density of states, and cyclotron masses were found with a relative error of less than $1 \%{ }^{20}$ The Dirac RAPW matrix used in the constant-energy-surface calculations was formulated by Loucks, ${ }^{21}$ and the basic computer subroutines were early versions of the ones given in Ref. 5 . The $\kappa$ summations included the $\kappa= \pm 10$ terms. Although the eigenvalues and the constant-energy surfaces were calculated by different programs using different formulations of the Dirac RAPW matrix, but using the same 44 basis, careful checks showed that the results were consistent to better than $0.1 \mathrm{mRy}$.

\section{RESULTS}

\section{A. Energy Bands (Gross Features)}

The relativistic energy bands of Pt were calculated along the lines of high symmetry by Mackintosh ${ }^{4}$ and are shown in Fig. 1. Figure 2 is an outline of our Rh bands, when spin-orbit splittings are neglected. No attempt was made to calculate eigenvalues over a broad energy range and at many points in the Brillouin zone, since we were only interested in energies near the Fermi surface. Energy eigenvalues far from $E_{F}$ were only calculated at the symmetry points and these eigenvalues are given in Table II, as they may be useful in calculations employing interpolation schemes.

The energy bands of the noble and transition metals consist near the Fermi level of a broad $s p$ band which is crossed by and hybridizes with a narrow $d$ band, and the essential parameters describing individual differences are the width and position of the virtual $d$ level. ${ }^{7,14}$

As a measure of the $d$ bandwidth, including relativistic effects, the energy separation between the uppermost $X_{7}^{+}$and the lower most $X_{6}^{+}$levels, or the separation between the uppermost $L_{4}^{+} L_{5}^{+}$ and the lowermost $L_{5}^{+}$levels, may be used. Either measure shows that, going from the $4 d$ to the $5 d$ metals, the $d$ bandwidth increases by a factor of 1. 34, whereas going from the 10-electron to 9electron metals it increases by the factor 1.22 (Table III). Of the latter $22 \%$ about $10 \%$ may be considered an effect of the decrease in lattice constants (Table I); but apart from this, the variations in $d$ bandwidth are attributable to variations in the width of the virtual $d$ level of the potential at one lattice site. ${ }^{7}$ At the same time as the $d$ bandwidth increases, i.e., in order $\mathrm{Pd}, \mathrm{Rh}, \mathrm{Pt}$, Ir, the $s p$ band moves towards lower energies relative to the $d$ band: For $\mathrm{Pd}$ and $\mathrm{Rh}$ the $p$-like $L_{6}^{-}$level falls above the uppermost $d$-like level $L_{4}^{+} L_{5}^{+}$; for Pt the $L_{6}^{-}$falls between the spin-orbit split $L_{4}^{+} L_{5}^{+}$and $L_{6}^{+}$levels, and eventually for Ir $L_{6}^{-}$falls below $L_{6}^{+}$.

Relativistic effects are quite important, especially for the heavier elements Ir and Pt. For the latter, Mackintosh ${ }^{4}$ also calculated the energy bands along the symmetry line $\Delta$ in the nonrelativistic case using the same Dirac-Slater potential as in the relativistic calculation. It was found that for Pt relativistic effects lowered the $6 s$-like $\Gamma_{1}\left(\Gamma_{6}^{+}\right)$level by $0.25 \mathrm{Ry}$, and a comparison with the values found from first-order perturbation of a nonrelativistic atomic calculation ${ }^{22}$ indicates that this lowering results from a large negative massvelocity term and a compensating positive Darwin term. For the atom, however, the perturbation yielded a total lowering of the $6 s$ level by no more than $0.13 \mathrm{Ry}$; a similar discrepancy was found in the $\mathrm{Re}$ calculation by Mattheiss, ${ }^{19}$ and is roughly speaking due to the renormalization to the WignerSeitz sphere of the $6 s$ wave function in the solid. The $6 p$ and $5 d$ orbitals are less concentrated at the core center and accordingly the mass-velocity lowering is somewhat smaller, and the Darwin 


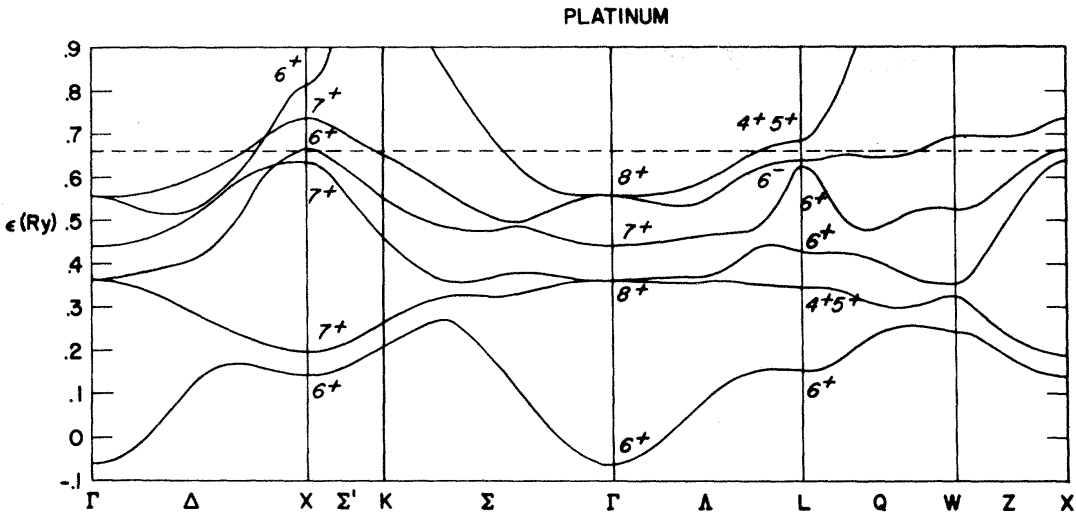

FIG. 1. Relativistic energy bands of Pt as calculated by Mackintosh (see Ref. 4). Double-group labeling has been used.

raising probably negligible, for such levels; the $6 p$-like $X_{4}$, was lowered by $0.18 \mathrm{Ry}$, and the $5 d$ like $X_{2}$ and $X_{3}$ levels by 0.16 , and $0.09 \mathrm{Ry}$, respectively. Apparently the $s p$ band is being broadened and lowered with respect to the $d$ bands by the mass-velocity and Darwin terms, but at the Fermi level, near the top of the $d$ band, the effects are relatively small for $\mathrm{Pt}$ and $\mathrm{Ir}$, and for $\mathrm{Rh}$ and Pd they are negligible. The $d$-band narrowing due to mass-velocity and Darwin corrections is compensated by the spin-orbit splittings at the top of the band $\left(X_{5}\right.$ and $\left.L_{3}\right)$, so that the overall effect of the relativistic terms on the total width of the $d$ band is small. However, for the shape of the Fermi surface and the velocities the spin-orbit splittings are important, even for $\mathrm{Rh}$ and Pd; this may be seen from Figs. 1 and 2.

The spin-orbit parameters $\xi_{l}(E)$, as derived from the Dirac-logarithmic derivations at the MTS (Appendixes), are shown as functions of energy in Fig. 3. For the resonating $d$ states these values are in agreement with the atomic spinorbit parameters given by Herman and Skillman, ${ }^{22}$ in the sense that the latter correspond to $\xi_{d}$ at an energy within the $d$ band; however, our parameters vary by $30 \%$ in the range of $d$-band energies. The spin-orbit parameters for the unbound $p$ states are again larger than the atomic values because the $5 p$ and $6 p$ wave functions spread far beyond the MTS and thus the normalization is affected. The RAPW values of the spin-orbit splittings in the $d$ band have been extracted from Table II and are given

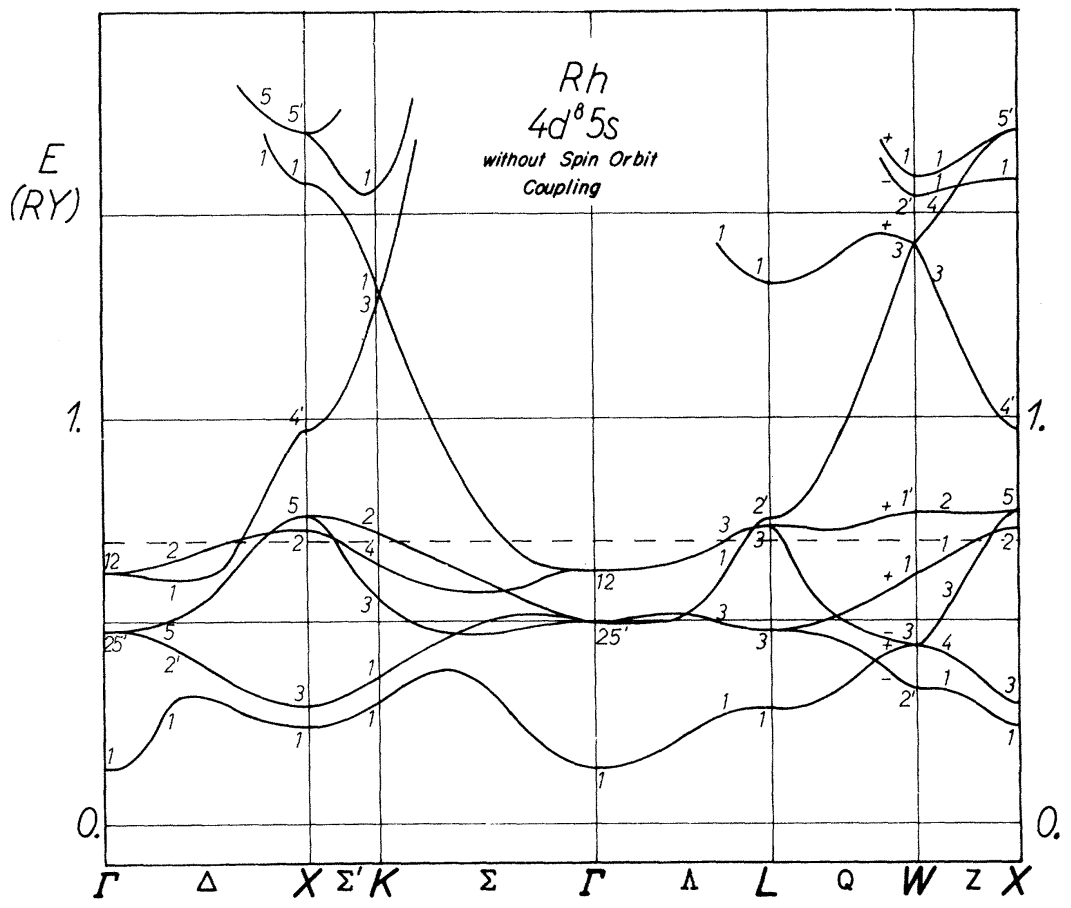

FIG.2. Outline of the energy bands of $\mathrm{Rh}$ when the spin-orbit coupling has been neglected. The energies have only been calculated at the points of high-symmetry and single-group labeling has been used. 
TABLE II. RAPW energy levels in fcc transition metals (energies in millirydbergs above MT0).

\begin{tabular}{|c|c|c|c|c|c|}
\hline & $\Gamma$ & $X$ & $K$ & $L$ & $W$ \\
\hline & 142 & 244 & 309 & 284 & 334 \\
\hline & 2486 & 296 & 370 & 470 & 442 \\
\hline $\mathrm{Rh}$ & 506 & 732 & 566 & 492 & 447 \\
\hline $4 d^{8} 5 s$ & 2632 & 761 & 649 & 732 & 615 \\
\hline \multirow{7}{*}{$E_{F}=701$} & & 780 & 729 & 745 & 770 \\
\hline & & 977 & 1293 & 757 & 1411 \\
\hline & & 1583 & 1347 & 1337 & 1441 \\
\hline & & 1691 & 1569 & & 1550 \\
\hline & & 1725 & & & 1594 \\
\hline & 43 & 161 & 215 & 187 & 237 \\
\hline & 2350 & 201 & 261 & 338 & 317 \\
\hline $\mathrm{Pd}$ & 375 & 547 & 422 & 364 & 327 \\
\hline $4 d^{10}$ & 2471 & 569 & 486 & 547 & 461 \\
\hline \multirow[t]{7}{*}{$E_{F}=557$} & & 591 & 548 & 561 & 578 \\
\hline & & 825 & 1089 & 628 & 1208 \\
\hline & & 1333 & 1157 & 1095 & 1236 \\
\hline & & 1525 & 1338 & & 1331 \\
\hline & & 1559 & & & 1380 \\
\hline & 29 & 227 & 303 & 249 & 338 \\
\hline & 2501 & 295 & 378 & 479 & 456 \\
\hline Ir & 570 & 823 & 600 & 548 & 469 \\
\hline $5 d^{7} 6 s^{2}$ & 2716 & 859 & 723 & 750 & 673 \\
\hline \multirow[t]{7}{*}{$E_{F}=790$} & & 928 & 837 & 824 & 888 \\
\hline & & 960 & 1285 & 868 & 1421 \\
\hline & & 1570 & 1373 & 1332 & 1426 \\
\hline & & 1640 & 1613 & & 1536 \\
\hline & & 1746 & & & 1732 \\
\hline & -60 & 141 & 205 & 150 & 236 \\
\hline & 2356 & 194 & 264 & 339 & 323 \\
\hline $\mathrm{Pt}$ & 436 & 631 & 456 & 417 & 349 \\
\hline $5 d^{9} 6 s$ & 2551 & 655 & 554 & 625 & 520 \\
\hline \multirow[t]{5}{*}{$E_{F}=654$} & & 729 & 650 & 628 & 687 \\
\hline & & 814 & 1094 & 677 & 1198 \\
\hline & & 1310 & 1164 & 1090 & 1228 \\
\hline & & 1476 & 1379 & & 1331 \\
\hline & & 1582 & & & 1511 \\
\hline
\end{tabular}

in Table IV. These values may be compared with the predictions of a $d$-band tight-binding model including the spin-orbit coupling as a perturbation, ${ }^{18}$ in which the values of the overlap integrals are determined from the actual energy eigenvalues and the spin-orbit parameter is taken as $\xi_{d}(E)$ from Fig. 3, with $E$ being the energy of the nonsplit levels:

In the Pauli representation the spin-orbit term is

$$
\frac{1}{2} \xi_{d} \vec{\sigma} \cdot \vec{I}=\frac{1}{2} \xi_{d}\left\{\begin{array}{ll}
l_{z} & l_{-} \\
l_{+} & -l_{z}
\end{array}\right\} .
$$

Therefore, to first order in $\xi_{d}$ the sixfold degenerate $\Gamma_{25}$ level, which has eigenfunctions derived from the spherical harmonics with $m= \pm 1$ and 0 , splits as if it were an $l=1$ level, and the splitting is $\frac{3}{2} \xi_{d}(E)$. The two $X_{5}$ states correspond to $m= \pm 1$; and the spin-orbit term between them is diagonal, so that the splitting is $\xi_{d}(E)$ to first order. The two $L_{3}$ splittings are of very different magnitude. However, as shown by Phillips and Mueller, ${ }^{23}$ when the axis of quantization is taken along [111], the upper $L_{3}$ level has almost solely $m= \pm 1$ character, with only a slight $m= \pm 2$ mixing, whereas the reverse is the case at the lower level. The tight-binding result is, that to first order the $L_{3}$ splittings are $A \xi_{d}(E)$ and $-(1+A) \xi_{d}(E)$, and $A$ is $0.65-0.75$, dependent on ratios between overlap integrals. The terms of higher order in $\xi_{d}$ are most easily obtained from the tight-binding matrices, factorized according to double-group representations ${ }^{18}$; here the energy dependence of $\xi_{d}$ must be neglected.

The result is that the agreement between the $d$ band spin-orbit splittings as calculated by the RAPW method and those found from tight-binding theory is better than 5\%, provided that for the firstorder terms the energy dependence of the muffintin spin-orbit parameter $\xi_{d}(E)$ is taken seriously. Otherwise errors of up to $6 \mathrm{mRy}$ for $\mathrm{Pd}$ and 20 mRy for Pt may occur.

At the points of high symmetry our RAPW spinorbit splittings for $\mathrm{Pd}$ and Pt agree perfectly with those recently obtained in a symmetrized RAPW calculation. ${ }^{24}$

\section{B. Fermi Surfaces and Density of States at $E_{F}$}

Owing to the similarity between the energy bands of all four metals, the Fermi surfaces of Rh and Ir containing 9 valence electrons per atom are very much alike, as are the 10-electron Fermi surfaces of $\mathrm{Pd}$ and $\mathrm{Pt}$.

\section{Rhodium and Iridium}

As each energy band is at least doubly degenerate (Kramers degeneracy) we shall call a $\vec{k}$ space region normal if four bands are occupied. Regions having more than four occupied bands are electron regions and those having three or less occupied bands are hole regions. A surface separating normal from electron regions or electron regions of different occupancy comprises part of an electron Fermi surface, and the analogous definition applies to the hole Fermi surfaces. Further we

TABLE III . Ratios of inverse $d$ bandwidths and total density of states at $E_{F}$.

\begin{tabular}{ccccccc}
\hline $\mathrm{Rh}$ & 1.25 & $\mathrm{Pd}$ & $4 d$ & $\mathrm{Rh}$ & 1.75 & $\mathrm{Pd}$ \\
1.31 & widths & 1.37 & & 1.35 & $N\left(E_{F}\right)$ & 1.41 \\
$\mathrm{Ir}$ & 1.19 & $\mathrm{Pt}$ & $5 d$ & $\mathrm{Ir}$ & 1.68 & $\mathrm{Pt}$ \\
9 & electrons & 10 & & 9 & electrons & 10 \\
\hline
\end{tabular}



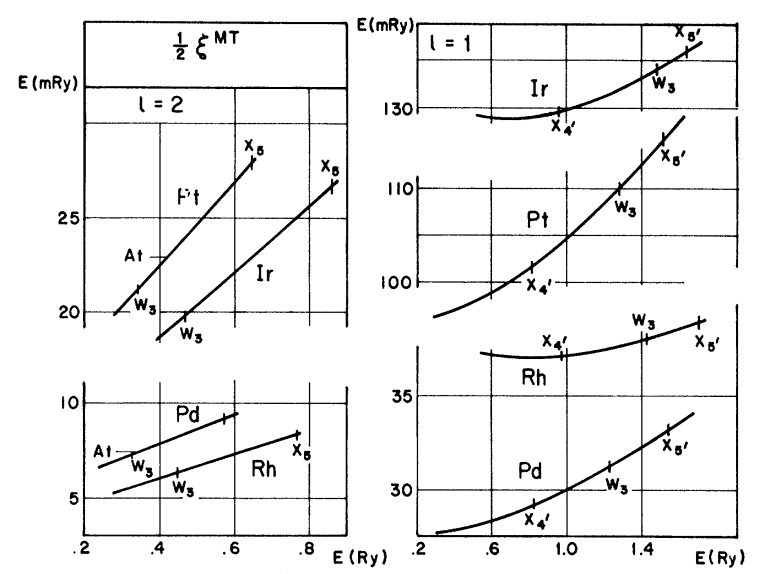

FIG. 3. Spin-orbit coupling parameters as derived from the Dirac-logarithmic derivatives by means of Eqs. (A2c), (A2f), and (C6). At indicate atomic values from Ref. 22 .

shall label sheets of Fermi surface by their band label, counting the bands in order of increasing energy, plus one or more letters labeling, respectively, the center for closed sheets, and the open direction for open sheets.

From the bands (Figs. 1 and 2) and the Fermisurface sections of Fig. 4 it may be seen that each metal has two closed electron surfaces $\Gamma 6$ and $\Gamma 5$, and two closed hole surfaces $X 4$ and $X 3$. Rh has an additional small hole surface $L 4$. The volumes in units of $\frac{1}{2}$ Brillouin-zone volume, i. e., the number of states per atom $n(E)$ and the partial density of states at the Fermi level $N\left(E_{F}\right)$, as derived from $n(E)$, may be found in Table I.

From the shape of the energy bands it seems apparent that the Fermi-surface sheets associated with the third, fourth, and fifth bands are primarily $d$-like, and that hybridization is important, especially for Ir, which has the largest bandwidth and the lowest-lying $s p$ band. We have not attempted to calculate wave functions, but a study of the variation in magnitude of the velocity on the different Fermi-surface sheets confirms the above. In both metals the velocities on $\Gamma 6$ are higher than on the other sheets; this is most pronounced for $\mathrm{Rh}$, and the $\Gamma 6$ state density is nearly as low as for a free-electron sphere of same dimension. Clearly, $\Gamma 6$ is the most $s p$-like sheet, but in order to decide whether the $s p$ or $d$ character dominates wave functions are needed. Near such symmetry lines, where hybridization does not occur for the bands in question, wave function characters may be exchanged: Along $\Delta$ (and $\Lambda$ ) the calculated velocities indicate a decrease of $s p$ character on $\Gamma 6$ and an increase on $\Gamma 5$. For Ir also $X 3$ has increased $s p$ character.
The Fermi levels are clamped with respect to the energies of states with high density, i.e., $d$ states [see Eq. (8)], and the $\Gamma 5$ volumes of $\mathrm{Rh}$ and Ir are almost equal. But the extent of decrease in the fifth band density of states, when going from $\mathrm{Rh}$ to Ir, is slightly larger than would be expected from the increase in $d$ bandwidth, because hybridization is more extensive for Ir. The Ir $\Gamma 6$ sheet is then more $d$-like, but nevertheless it may be seen that its density of states is smaller than the density of states of $\Gamma 6$ for Rh. This is because the size of $\Gamma 6$ is being reduced as spin-orbit coupling raises the energies of the sixth band near the (110) planes from $\Lambda$ to $\Delta$. Due to hybridization and spin-orbit coupling, we therefore obtain the somewhat unexpected result that, in spite of a lower-lying $s p$ band, Ir has less $\Gamma 6$ electrons than Rh.

However, the ratio between the $\mathrm{Rh}$ and Ir total density of states is only $3 \%$ greater than the ratio of the inverse $d$ bandwidths (Table III), so apparently the difference in $d$-band position and strength of spin-orbit coupling merely changes the distribution of density of states, i. e., $|v(\overrightarrow{\mathrm{k}})|^{-1}$, from one sheet to another, and the integral over the entire Fermi surface remains nearly constant.

\section{Palladium and Platinum}

In these metals normal $\vec{k}$ space regions are defined as those having five occupied bands. Each metal has a closed electron surface $(\Gamma 6)$ which is larger, but of the same shape as the corresponding Rh.and Ir surfaces (Figs. 4 and 5). For Pd and $P t$ the fifth band Fermi surface (XW5) is now an open hole surface extending in the [100] directions and of primarily $d$ character (Fig. 6). The $X 3$ holes have disappeared, but holes still exist in the fourth band inside $X 4$, which is $d$-like. Moreover, we found a very small hole pocket (L5)

TABLE IV. RAPW spin-orbit splittings for fce transition metals (energies in millirydbergs).

\begin{tabular}{|c|c|c|c|c|}
\hline & $\Gamma$ & $X$ & $L$ & $W$ \\
\hline & $25^{\prime} \rightarrow 7^{+}+8^{+}$ & $5 \rightarrow 6^{+}+7^{+}$ & $\begin{array}{l}3 \rightarrow\left(4^{+}+5^{+}\right)+6^{+} \\
3 \rightarrow\left(4^{+}+5^{+}\right)+6^{+}\end{array}$ & $3 \rightarrow 6+7$ \\
\hline $\begin{array}{l}\mathrm{Rh} \\
4 d^{8} 5 s\end{array}$ & 20 & 19 & $\begin{array}{r}-22 \\
13\end{array}$ & 5 \\
\hline $\begin{array}{l}\mathrm{Pd} \\
4 d^{10}\end{array}$ & 25 & 22 & $\begin{array}{r}-26 \\
14\end{array}$ & 10 \\
\hline $\begin{array}{l}\text { Ir } \\
5 d^{7} 6 s^{2}\end{array}$ & 69 & 69 & $\begin{array}{r}-69 \\
44\end{array}$ & 13 \\
\hline $\begin{array}{l}\mathrm{Pt} \\
5 d^{9} 6 s\end{array}$ & 80 & 74 & $\begin{array}{r}-78 \\
52\end{array}$ & 26 \\
\hline
\end{tabular}




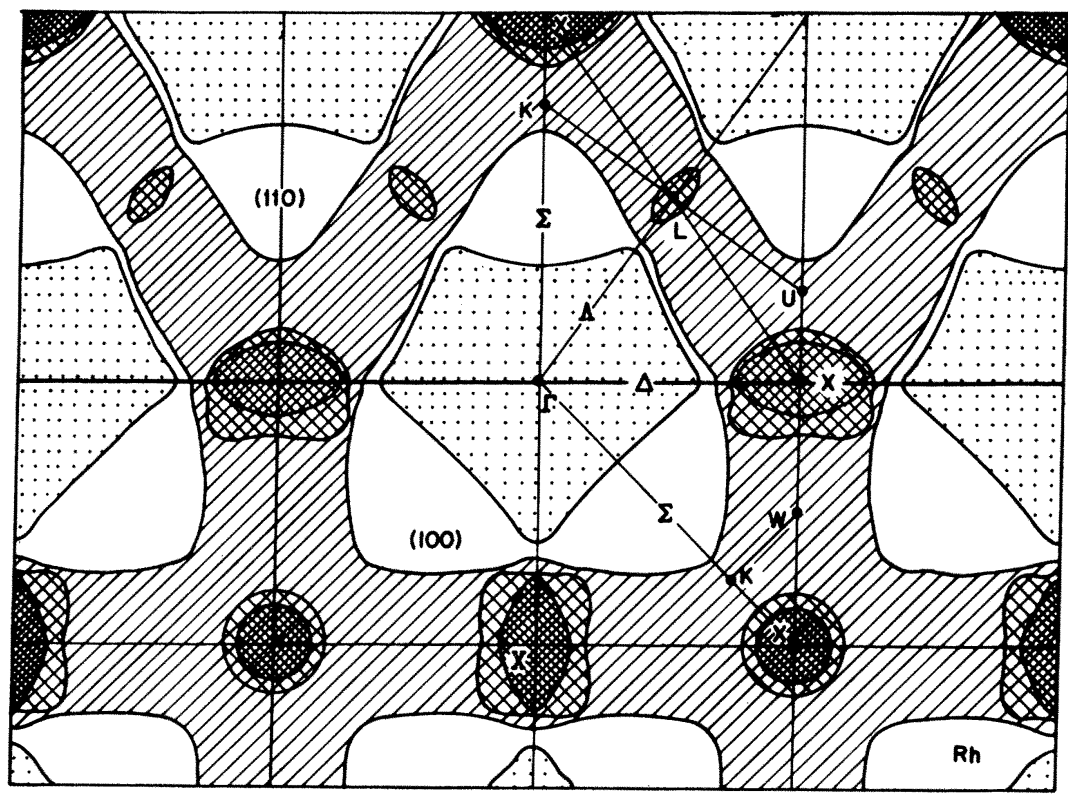

FIG. 4. Cross sections in the central (100) and (110) planes of the Fermi surface for Rh (top) and Ir

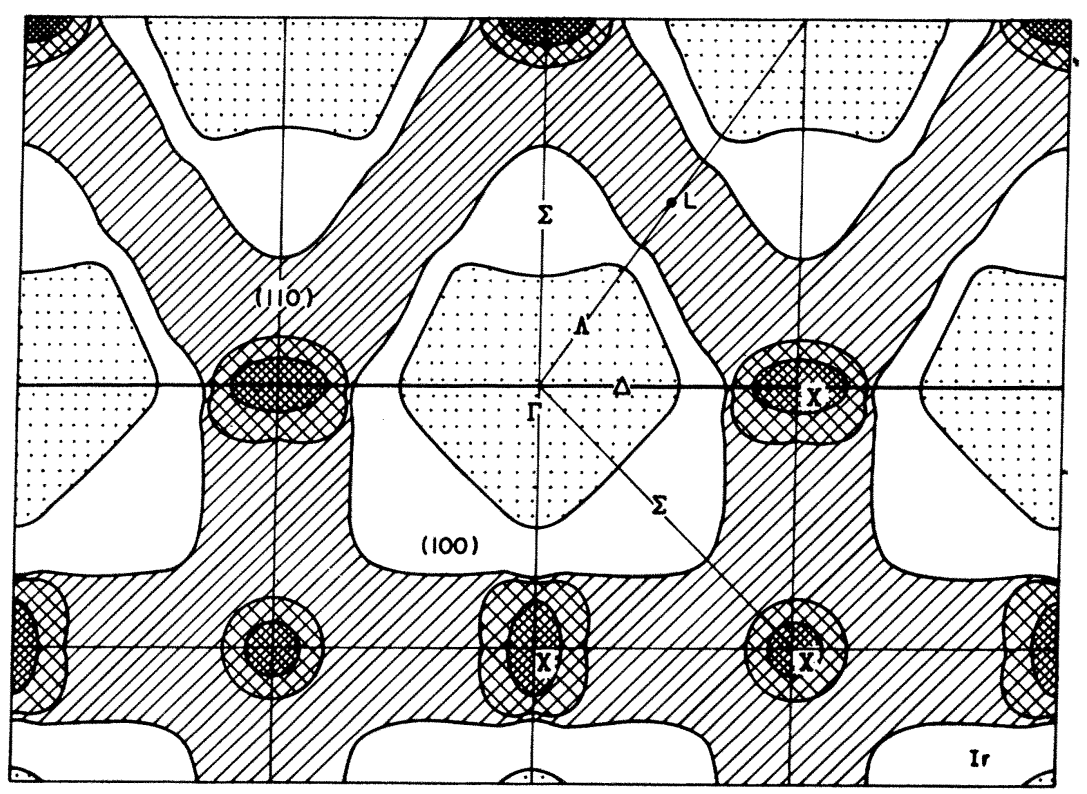
(bottom). Normal regions (Sec. III B) are singly cross hatched, and the periodic zone scheme has been employed (see Fig.9 and Sec. III C).

in Pd, but its existence is dubious.

Due to the higher-lying Fermi level, hybridization is less important for the 10-electron Fermi surfaces of Pd and Pt than for the 9-electron Fermi surfaces of $\mathrm{Rh}$ and Ir, and again hybridization is less important for the lighter element Pd. As seen from Table I the $X W 5$ partial density of states is very high, especially for $\mathrm{Pd}$, which has the most narrow $d$ bands and an almost purely $d$-like $X W 5$. Here again the more severe $s p-d$ hybridization for the heavy element causes the ratio between the Pd and Pt partial densities for $X W 5$ to be somewhat greater than might be expected from the ratio of $d$ bandwidths. For the same reason there are more $\Gamma 6$ electrons in $\mathrm{Pt}$ than in Pd.

The Fermi velocities in the (110) plane of $\Gamma 6$ are shown in Fig. 7, and the difference between the $\mathrm{Pd}$ sheet and the severely hybridized Pt sheet may clearly be seen. Near $\Delta$ and $\Lambda$ the Pt $\Gamma 6$ is highly $d$-like and the $X W 5$ correspondingly more $s p$-like, whereas the character of each Pd Fermi-surface sheet is more uniform.

The velocities at $X W 5$ (Fig. 6) vary greatly. Even for $\mathrm{Pd}$, where the $d$ character dominates strongly everywhere, the velocities are $0.27,0.18,0.18$, and 0.022 a.u. at $\Delta, \Sigma, L W(Q)$, and $L X$, respec- 

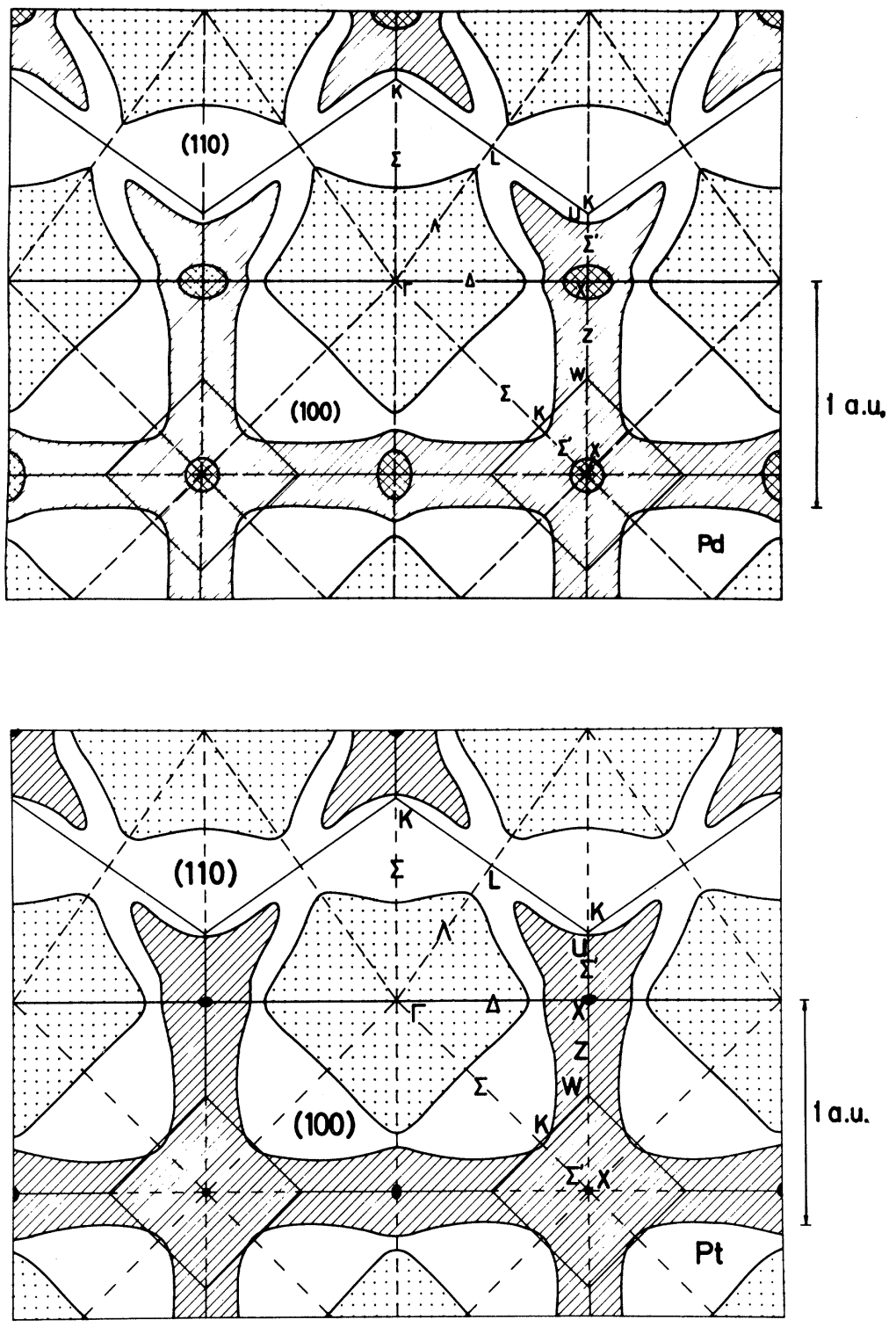

FIG. 5. Cross sections in the central (100) and (110) planes of the Fermi surface for Pd (top) and Pt (bottom). Normal regions are blank. tively. For Pt the numbers are 0.93, 0.29, 0.23, and $0.033 \mathrm{a} . \mathrm{u}$. This may also be seen from the Pd constant-energy contours in Fig. 8. The extremely low velocities at the "fins" are due to a saddlepoint in $E(\overrightarrow{\mathrm{k}})$ close to $L$ on the line $L X$ and at an energy just a few mRy's below the Fermi energy (Fig. 6). A cylindrical approximation to $X W 5$, as obtained by rotating the constant-energy contours of the (001) plane around the [100] cylinder axis (Fig. 8), and thus cutting off the fins, would yield less than half the fifth band density of states at the Fermi level for both Pt and Pd.

The ratio between the total density of states for $\mathrm{Pd}$ and $\mathrm{Pt}$ is $3 \%$ greater than the ratio between the inverse $d$ bandwidths, exactly as for Rh and Ir
(Table III). For Pd and Pt we have moreover seen how the $s p$ versus $d$-band position affects the distribution of Fermi velocities in $\overrightarrow{\mathrm{k}}$ space.

\section{Constant - Energy Surfaces and Partial Density of States of the Rh and Pd Fifth Bands}

Various experiments (Sec. IV A) and band-structure calculations on fcc transition and noble metals $\mathrm{s}^{8,18,25,26}$ indicate the existence of a pronounced peak in the density of states near the top of the $d$ band at an energy slightly below the Fermi level of $\mathrm{Pd}$ and $\mathrm{Pt}$. In a rigid-band description the peak occurs near Pd for a PdRh system and near Pt for a PtIr system. In Sec. III B we saw that the major contribution to $N\left(E_{F}\right)$ came from the fifth 


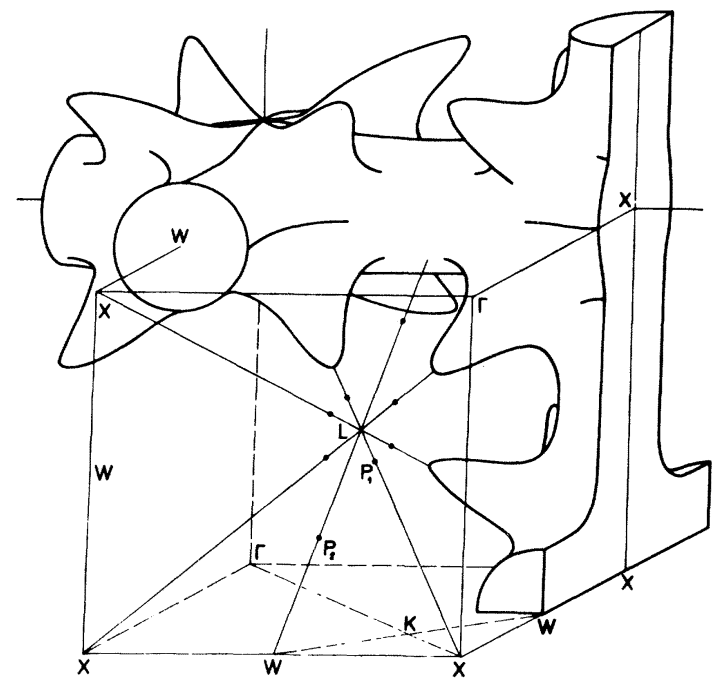

FIG. 6. Sketch of the open hole surface $X W 5$ of the Pd or Pt Fermi surface in the periodic zone scheme. The part of reciprocal space shown, and its connection with the Brillouin zone, may be found in Fig. 9. The critical points $P_{1}$ and $P_{2}$ have also been indicated (Sec. III C).

band; moreover, the fifth band Fermi-surface sheets of $\mathrm{Rh}$ and Ir, i.e., $\Gamma 5$, have a topology quite different from that of the Pd and Pt $X W 5$ sheets (Figs. 4 and 5). We therefore anticipate that the $N(E)$ peak arises from this band and that the shape of the peak is determined by van Hove singularities.

Most other theoretical estimates of $N(E)^{8,25,26}$ have employed the histogram technique, according to which eigenvalues are calculated at points uniformly distributed over the entire irreducible zone and thereafter sorted according to energies. For the special purpose of studying fine details in $N(E)$ over a narrow energy range this technique has the disadvantage that all computations for points outside the volume between the two constant-energy surfaces, which bracket the energy range, are wasted. Moreover, the reproduction of van Hove singularities is usually poor. The method of tracing constant-energy surfaces is, on the other hand, selective in $E$ and $\overrightarrow{\mathrm{k}}$, but it forces topological thinking.

The fcc structure has a bcc reciprocal lattice, which is shown by the $\Gamma$ points in Fig. 9(a), and in the periodic zone scheme the group of $E(\overrightarrow{\mathrm{k}})$ consists of the covering operations of the bcc lattice. When, in a rigid-band language, the energy is raised from the Fermi level of $\mathrm{Rh}$ towards that of Pd the closed constant-energy surfaces $\Gamma 5$ expand until neighboring sheets make contact at a saddlepoint of type $P_{2}{ }^{27}$ in $E(\overrightarrow{\mathrm{k}})$ situated $25 \mathrm{mRy}$ above the Fermi level of $\mathrm{Rh}$ and about half-way between the symmetry points $L$ and $W$ on the twofold axis $Q$; thereafter a multiply connected surface $L X W 5$ is formed (Fig. 9). Increasing the energy further the necks at $P_{2}$ get wider, and we may start to think of those regions having four occupied bands as hole regions (shaded in Fig. 9) and of the five band regions as normal regions (unshaded). The topological difference between $L X W 5$ and the Fermi-surface sheet $X W 5$ of $\mathrm{Pd}$ is that the former is connected along $L X$. This connectivity breaks at a $P_{1}$ saddlepoint just $2 \mathrm{mRy}$ below the Fermi level of $\mathrm{Pd}$. The resulting $X W 5$ and $L 5$ sheets are not related by symmetry, and the small $L 5$ pocket probably disappears just below the Pd Fermi level. ${ }^{28}$ The "fins" of the Pd Fermi surface are the remains of the connections along $L X$.

It may be noted that the $\Gamma 5$ sheet breaks through the hexagonal face (111) of the Brillouin zone before the $P_{2}$ contact, ${ }^{29}$ therefore the conventional irreducible zone [Fig. 9(b)] is not a useful frame for the tracing of these surfaces. We may define an irreducible zone as a simply connected region which under the space group [of $E(\vec{k})]$ maps one to one onto the entire $\overrightarrow{\mathrm{k}}$ space, i.e., an irreducible zone can have no symmetry element (rotation axis

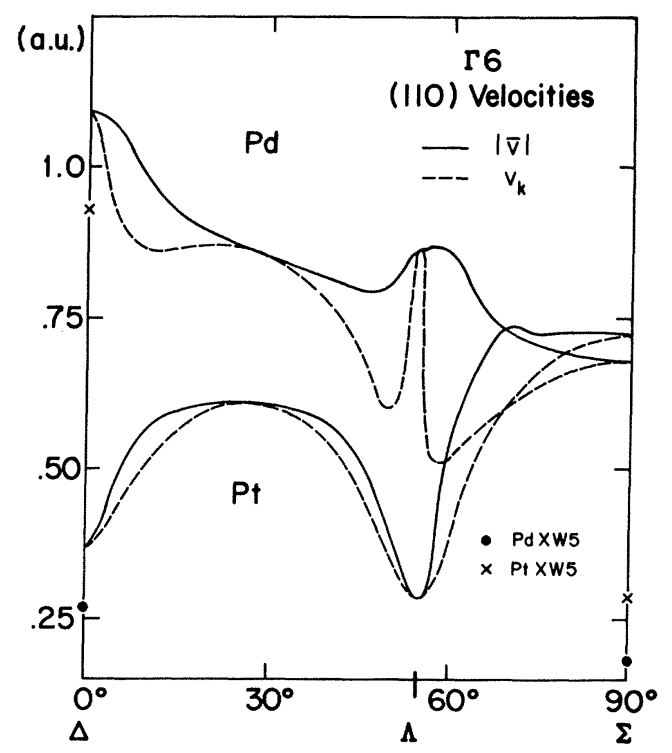

FIG. 7. Magnitude and radial component of velocities in the (110) plane on the $\Gamma 6$ Fermi-surface sheet of Pd and Pt. The radii may be found in Fig. 5 as those bounding the dotted region. In order to show the effect of hybridization the velocities on $X W 5$ along $\Delta$ and $\Sigma$ have been marked. Comparison with experimental velocities for Pt may be found in Fig. 2 of Ref. 62 (Sec. IV B). 


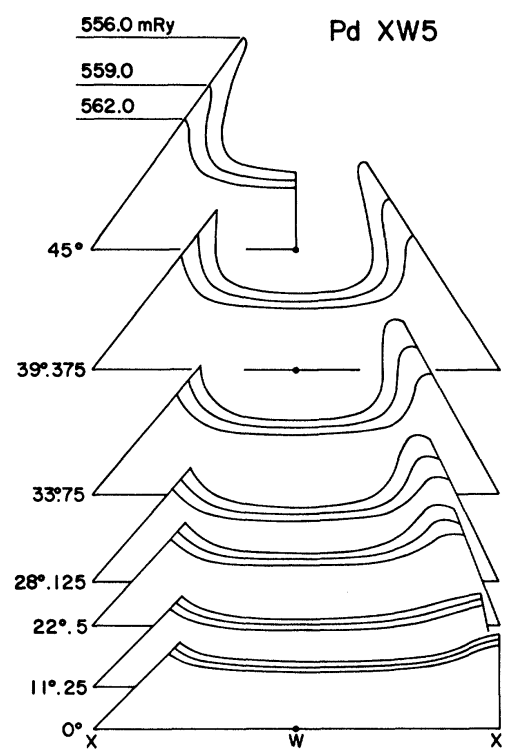

FIG.8. Computer-traced constant-energy contours for $X W 5$ of Pd (Fig。6) in the irreducible zone of Fig. 9 (b) (Sec. III C). $[X W]$ is the common axis of the sections, which are labeled by their respective angles with the (100) plane; the $45^{\circ}$ section is in the $(X W X L)$ plane [Fig。 $9(\mathrm{~b})$ ]。 The Fermi energy is $556.9 \mathrm{mRy}$ 。

or reflection plane) in its interior, and any of its boundaries is either a reflection plane or a surface containing a rotation axis. The latter surface must be invariant with respect to the rotation, but is otherwise arbitrary. Returning now to the fcc structure and Fig. 9 the twofold axis $Q$ allows us to turn the (111) boundary plane of the conventional $\frac{1}{48}$ Brillouin zone around $Q$ by any angle. Figure 9(b) shows the conventional (111) irreducible zone and the (110) irreducible zone, which is the useful zone for $\Gamma 5$ sheets, and also for $X W 5$ sheets. The (100) irreducible zone [Fig. 9(d)] is useful for $X W 5$ sheets, since these lie entirely in the $X W W L$ cube; further, it is convenient for numerical $\vec{k}$ space integrations.

In order to find the accurate energies and $\vec{k}$ space locations of the $P_{2}$ and $P_{1}$ saddlepoints the fifth bands of $\mathrm{Rh}$ and $\mathrm{Pd}$ were traced out along $\Lambda(L \Gamma), Q(L W)$, and $L X$. The Pd band is shown in Fig. 10, and the $\mathrm{Rh}$ band is very similar except for a scale factor of 1.25. The Rh $\Gamma 5$ surfaces at the energies $E\left(P_{2}\right)$ and $0.7 \mathrm{mRy}$ below $E\left(P_{2}\right)$ were traced out and so were six Pd $X W 5$ surfaces, separated by $1.5 \mathrm{mRy}$, at and above $E\left(P_{1}\right)$. From there the number $n$ and density $N$ of states of the fifth band were found at and near the critical points. The results may be found in Table V. In the interval between the $\mathrm{Rh}$ and Pd Fermi energies, no other singularities exist in the fifth band than those mentioned $\left(P_{2}\right.$ at $Q, P_{1}$ at $L X$, and the maximum at $L$ ), and of these only the two saddle points are important. The density of states is therefore a smooth function of energy between these singularities, and knowing the integral of $N_{5}(E)$, i. e., $n_{5}$, over the smooth regions, we can reliably sketch $N_{5}(E)$ over the entire interval from the Fermi energy of $\mathrm{Rh}$ to that of $\mathrm{Pd}$. The contribution from the sixth band was found for Rh at $E\left(P_{2}\right)$ by constant-energy contour tracing; the contributions from the third and fourth bands are small and have been found from extrapolation from the Fermi-surface results, using the parabolic-band approximation. Again, van Hove singularities contributed by band maxima or minima were found to be much less important than those contributed by saddle points. In Fig. 11 we show for RhPd the rigidband density of states $N(n)$, which is the $n$-weighted mean between the Pd and Rh density of states. From here it may be seen that it is the combined effect of the two saddle points $P_{1}$ and $P_{2}$, rather than one or neither of these, which is responsible for this density-of-states peak, probably the highest of the entire $904{ }_{l .}{ }^{30}$

The partial density of states for the fifth band of $\mathrm{Pd}$ in the interesting energy region around $E\left(P_{1}\right)$ and $E_{F}$ was determined from the six above-mentioned constant-energy contours, each consisting of 100 points in the $\frac{1}{48}$ Brillouin zone (Fig. 8). Knowing the value of $E\left(P_{1}\right)$ the volumes $n_{5}(E)$ were fitted by least squares to a power expansion in $\Delta E$ $=E-E\left(P_{1}\right)$ containing the van Hove term $\Delta E^{3 / 2}$, and the density of states was obtained by differentiation; the results may be found in Table VI and Fig. 12. For the given band, the difference between any of the six calculated volumes was more accurate than $10^{-4}$ states per atom. The rms deviation of the power expansion was $0.8 \times 10^{-4}$ states per atom, whereas the deviation of an integral power expansion was much greater, thus indicating the importance of the van Hove term. Also the total density of states was fitted to the power expansion, since every singularity of the sixth and fourth bands is well outside the energy region here considered. ${ }^{31}$

Finally, we would like to point out that the topological features discussed in this section are very relevant to ferromagnetic $\mathrm{Ni}$, since the Fermi-surface sheet of the fifth minority spin band is probably similar to a Rh $\Gamma 5$ constant-energy surface containing approximately $8+1.1$ electrons/atom.

\section{COMPARISON WITH EXPERIMENTS}

A. Electronic Specific Heat and Susceptibility Data

The coefficient of the linear term of the low-temperature specific heat is, in the one-electron the- 

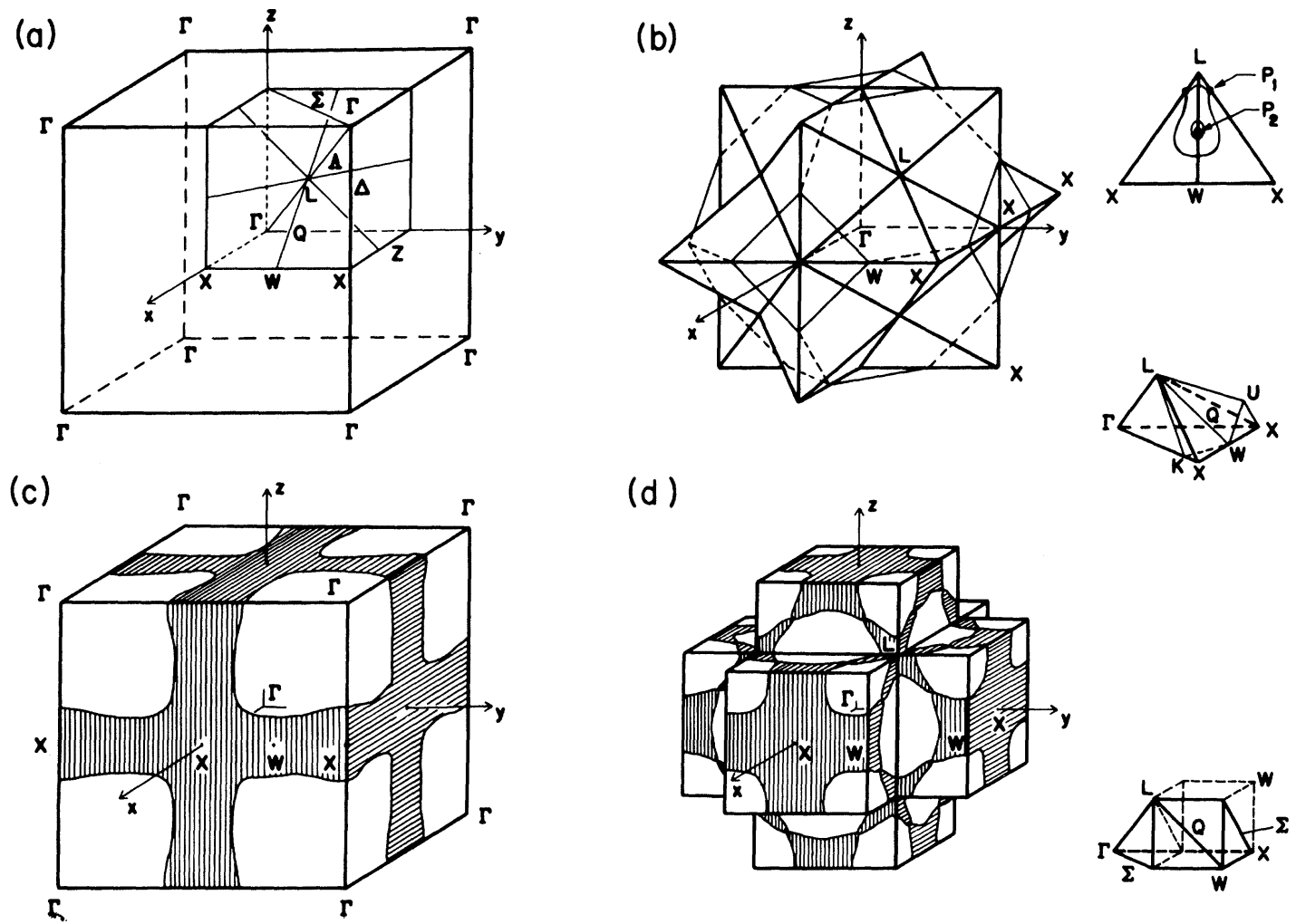

(d)

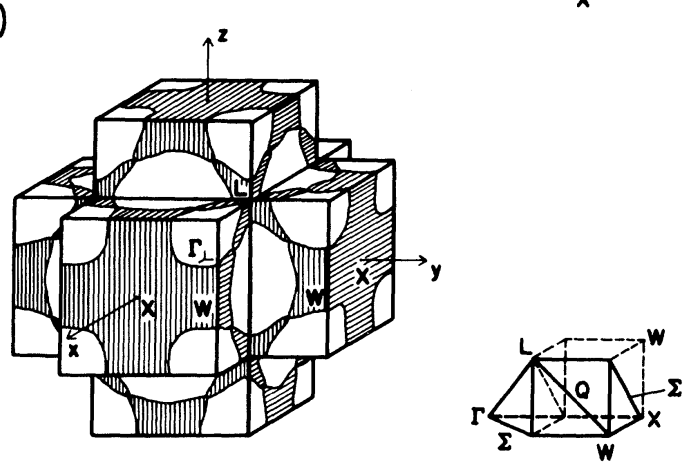

FIG. 9. Various unit cells and irreducible zones in reciprocal space of the fcc structure and contours of the critical $\Gamma 5-L X W 5$ surfaces for $\mathrm{Rh}$ and $\mathrm{Pd}$. (a) bcc unit cell containing two reciprocal-lattice points $\Gamma$. Various symmetry points and lines have been shown in one of the octants. (b) Unit cells containing one reciprocal-lattice point, the Brillouin zone in weak lines, and the " $X$-star" in heavy lines. The corresponding irreducible zones (111) and (110) have been shown in the lower inset: the former has five, but the latter only four faces. Both irreducible zones have the twofold axis $Q[L W]$ on their boundary. The boundaries of the $X$ star consist of the $48(X W X L)$ planes, of which one is shown in the upper inset, and these planes contain the critical points $P_{2}$ and $P_{1}$ of the fifth band in Rh and Pd. The axis of the saddle point at $P_{2}$ is orthogonal to the $(X W X L)$ plane so that the $X$ star has the same topology as the $\Gamma 5$ surface. The axis of the $P_{1}$ saddle point is $[L X]$, and the illustrated energy contours correspond to $E \gtrsim\left(P_{2}\right)$ and $E=E\left(P_{1}\right)$. (c) Contours of the critical $\Gamma 5-L X W 5$ surface on the boundaries of the unit cell of (a). The electron region of the fifth band is blank, whereas the hole region is cross hatched. (d) Unit cell containing one reciprocal-lattice point and its irreducible zone. The (100) planes through $L$ and $W$ are orthogonal to the $(X W X L)$ planes of (b) with traces $Q[L W]$. The $P_{2}$ contact on $Q$ between neighboring $\Gamma 5$ electron regions may be seen. A cubic mesh for numerical integration or interpolation is conveniently embedded in this unit cell, as it may be divided into 32 equally sized boxes: 24 of type $X W W L$, in which one diagonal plane is a mirror plane, and 8 of type $\Gamma L$, in which all three diagonal planes are mirror planes. In each type of box a cubic mesh is mapped onto itself by the reflections in the diagonal planes, so that the function to be treated, e.g., $E(\vec{k})$, need only be calculated in a number of points corresponding to $\frac{1}{48}$ Brillouin-zone volume. Further, the two types of boxes may be stacked by using the three mirror faces at $X$ and $\Gamma$ and the twofold axes $Q[L W]$.

ory, $\gamma_{\text {band }}=\frac{1}{3} \pi^{2} k_{B}^{2} N\left(E_{F}\right)$, and our theoretical values have been compared with the experimental results of Budworth, Hoare, and Preston ${ }^{32}$ (Rh), Dixon et al. ${ }^{33,34}$ (Ir), Veal and Rayne ${ }^{35}(\mathrm{Pd})$, and Shoemake and Rayne ${ }^{36}(\mathrm{Pt})$ in Table VII.

The enhancement factors include the effects from the electron-phonon interaction and from spin fluctuations. Of these four metals Ir is the only observed superconductor, and from experimental data on its transition temperature, Debye temperature and the isotopic shift of the transition temperature of neighboring elements McMillan ${ }^{37}$ estimated the electron-phonon enhancement to be 1.34. This value is in excellent agreement with the value in Table VII, since for Ir no significant contribution from spin fluctuations is expected. The enhancement factors increase in the same order as does the density of states, i.e., Ir, Rh, Pt, and $\mathrm{Pd}$, but the increase is small and the Pd enhancement is certainly not outstandingly large, in contrast to the predictions of simple spin-fluctuation theory.

The electronic specific-heat coefficients of $\mathrm{RhPd}$ and PdAg alloys have been measured by Budworth, Hoare, and Preston ${ }^{32}$ and by Hoare and Yates, ${ }^{38}$ respectively, and the enhancement over the rigidband density of states in Fig. 11 is displayed in the 

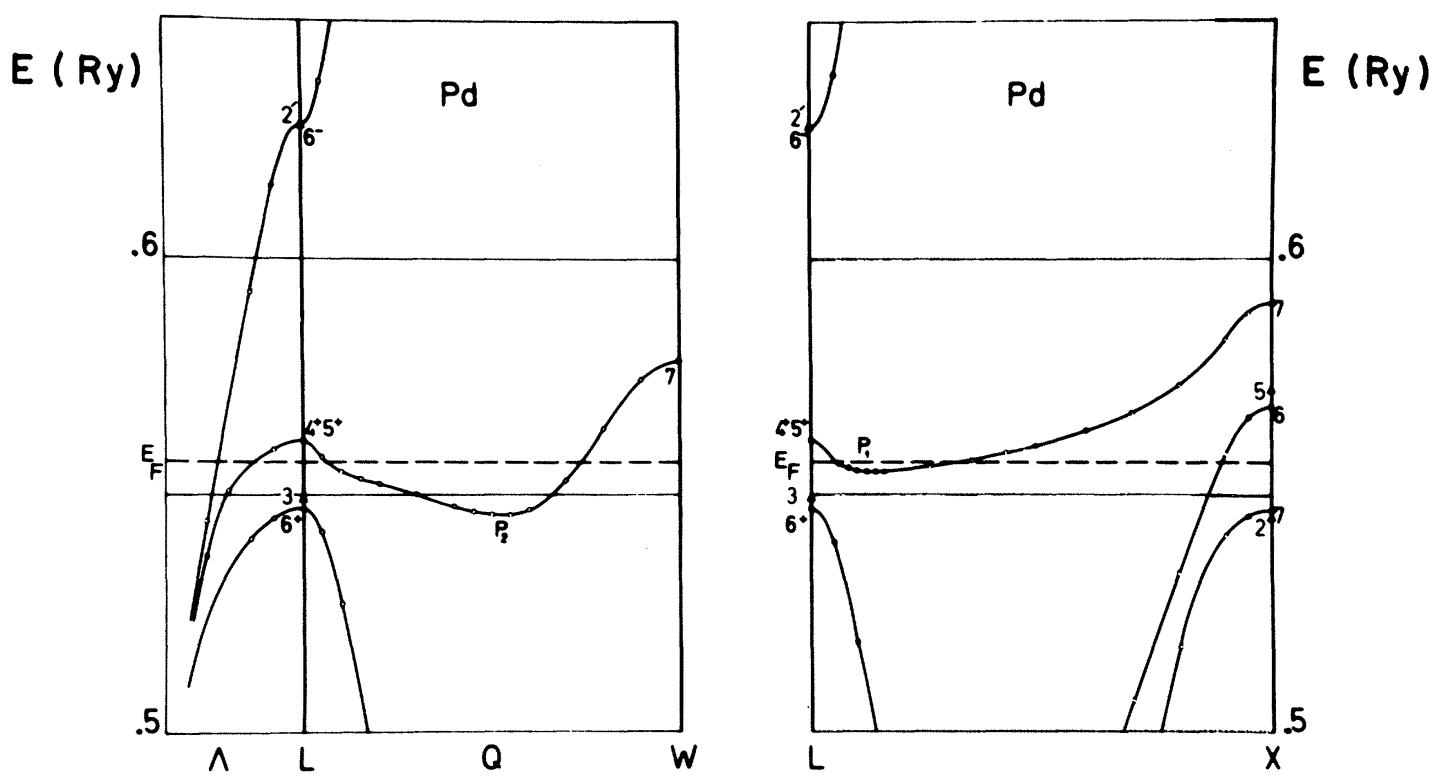

FIG. 10. Extremely flat parts of the fifth band of Pd showing the $P_{1}$ and $P_{2}$ saddle points [see also inset of Fig. $9(\mathrm{~b})$ and Refs. 28 and 31].

lower half of the same figure. The drop of the enhancement in the broad medium range of concentrations is probably due to the failure of the rigidband approximation, so in the disordered system the structure in the density of states is smeared out considerably. ${ }^{39}$ The experimental peak in the electronic specific-heat coefficient occurs at about $\mathrm{Rh}_{0,05} \mathrm{Pd}_{0,95}$, whereas the peak in the rigid-band density of states is at about $\mathrm{Rh}_{0,20} \mathrm{Pd}_{0.80}$; the $P_{1}$ singularity, however, was found at $\mathrm{Rh}_{0,07} \mathrm{Pd}_{0,93}$. It may be noted that photoemission and reflectivity data $^{40}$ confirm that the peak in the bare density of states for Pd is about $7 \mathrm{mRy}$ below the Fermi level, which in the rigid-band model corresponds to $\mathrm{Rh}_{0.20} \mathrm{Pd}_{0.80}$. Another departure from the rigidband model is the discontinuity in slope of $\gamma_{\text {exp }} / \gamma_{\text {band }}$ when going from a $\mathrm{Rh}$ to an $\mathrm{Ag}$ impurity in Pd.

The electronic specific-heat and susceptibility measurements on $\mathrm{RhNi}$ of Bucher et al. ${ }^{41}$ show how the susceptibility $\chi$, and possibly also $\gamma$, tend to diverge at the ferromagnetic instability. Taking our RhPd density of states as the appropriate band value, the rise in $\gamma_{\text {exp }} / \gamma_{\mathrm{b} \text { and }}$ towards instability is seen to set in abruptly just beyond $55 \% \mathrm{Ni}$ (Fig. 11). At $55.5 \%$ Ni the $\gamma$ enhancement is 1.9 , whereas the $\chi$ enhancement, i.e., the Stoner factor $D$, is 25, and at $63 \%$, just before the onset of ferromagnetism, the numbers are 2.3 and 80 , respectively. Whereas the initial (i.e., below 55\%) increase in $\gamma_{\text {exp }} /$ $\gamma_{\text {band }}$ may simply be due to the approximation of using RhPd instead of RhNi "bands" (the $d$ bands of paramagnetic Ni are more narrow than those of $\mathrm{Pd}$ ), the steep rise beyond $55 \%$ is probably a real physical effect. But whether this is due to spin fluctuations or to a magnetic contribution to $\gamma$ caused by an inhomogeneous $\mathrm{Ni}$ distribution ${ }^{42}$ is not clear at present.

In addition to the paramagnetic spin susceptibility, $\chi_{P}=\mu_{B}^{2} N\left(E_{F}\right)$, from $d$ and $s$ bands the susceptibility of a transition metal includes orbital paramagnetism, Landau diamagnetism, and core diamagnetism. $^{43}$ For $\mathrm{Pt}$ it has been estimated ${ }^{43}$ that the observed susceptibility is in fact equal to the $d$-spin susceptibility because the other terms tend to cancel, and for $\mathrm{Pd}$ the exchange enhancement of the $d$-spin susceptibility is so large that the latter term overshadows all other contributions. ${ }^{44}$ For Ir and $\mathrm{Rh}$ such simplifications are probably not valid.

The experimental values of the low-temperature low-field susceptibility of Foner and $\mathrm{McNiff}^{45}$ are $6.9 \times 10^{-6} \mathrm{emu} / \mathrm{g}$ and $1.08 \times 10^{-6} \mathrm{emu} / \mathrm{g}$ for $\mathrm{Pd}$ and $\mathrm{Pt}$, respectively. Accordingly, we find the enhancement $D$ over the $d$-spin susceptibility to be 9. 4 for Pd and 3. 8 for Pt. $^{46}$

The variation with energy of the band density of states brings about a dependence on temperature $T$ and magnetic field $B$ of the spin susceptibility. ${ }^{47}$

If the density of the states $N(E)$ is analytical in the relevant range around the Fermi level we have, to lowest order in temperature and magnetization, $\sigma^{48}$ :

$$
\begin{array}{ll}
1 / \chi_{T}=1 / \chi_{0}-\eta\left(\pi k_{B} T\right)^{2} / 6 \chi_{P} & \text { for } B \rightarrow 0, \\
B / \sigma=1 / \chi_{0}-\nu\left(\mu_{B} \sigma\right)^{2} / 6 \chi_{P}^{3} & \text { for } T \rightarrow 0,
\end{array}
$$

where $\chi_{0}=D \chi_{P}=\mu_{B}^{2} D N\left(E_{F}\right)$,

$$
\eta=\left[N^{\prime \prime} / N-\left(N^{\prime} / N\right)^{2}\right]_{E_{F}} \text {, }
$$




$$
\nu=\left[N^{\prime \prime} / N-3\left(N^{\prime} / N\right)^{2}\right]_{E_{F}} .
$$

From our detailed expression for the Pd density of states (Table VI) we have obtained $\eta$ and $\nu$ for $\mathrm{Pd}$ and, using the rigid-band model, for dilute PdRh and PdAg alloys (Fig. 13). For Pd and PdAg our values of $v$ and $\eta$ are consistent with the measurements of Foner and co-workers, ${ }^{45,49}$ but the effects are of the same order of magnitude as the experimental error. A good indication of the accuracy of the shape of our density-of-states curve is that the observed upturn in $\chi$ versus $T$ disappears at an Ag concentration between 2 and $3 \%$ in excellent agreement with the zero of our $\eta$; but this provides no check on the energy scale of our $d$ band. As may be seen from Fig. 13, $\nu$ and $\eta$ increase rapidly with $\mathrm{Rh}$ concentration, and at the same time the range of validity of Eqs. (2) and (3) becomes more and more limited. At the van Hove singularity $\nu$ and $\eta$ diverge, and the second-order expansions are not valid. We have therefore obtained ${ }^{50}$ the general $B / \sigma$ curves avoiding the usual Taylor expansion of $N(E)$, and have compared with the high-field mea-

TABLE V. Number of states $n$ and density of states $N$ for Rh and $\mathrm{Pd}$ at the van Hove singularities $P_{1}$ and $P_{2}$ of the fifth band. The underlined numbers have been calculated directly by computer tracing of constant-energy surfaces, or energy bands. The number and density of states of the third and fourth bands have been obtained by extrapolation from the corresponding values at the Fermi energy of Rh or Pd using the energy of the band maxima and the parabolic-band approximation. Bracketed numbers have been obtained by scaling $d$-band values by 1.25 , which is the ratio of $\mathrm{Rh}$ to Pd bandwidth. The value of $N_{5}$ half-way between $E\left(P_{1}\right)$ and $E\left(P_{2}\right)$ was obtained from Simpson's integration rule. Energies are in mRy above MT0, numbers of states are in states in excess of 8 per atom and densities of states are in (states/atom)/Ry.

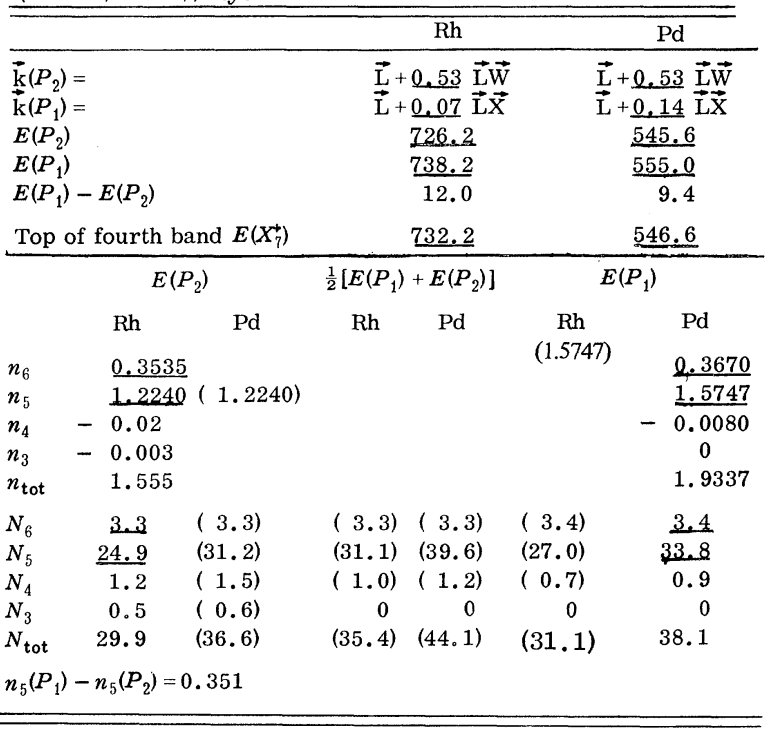

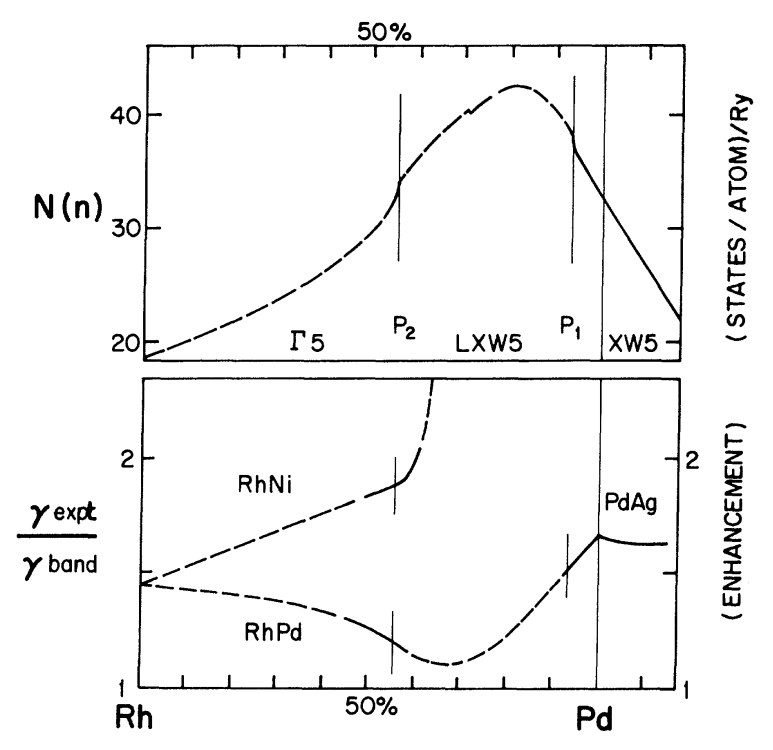

FIG. 11. Rigid-band density of states and enhancement of the electronic specific-heat coefficient $\gamma$ for RhNi, $\mathrm{RhPd}$, and dilute PdAg alloys. The fifth band singularities are $P_{1}$ and $P_{2}$, and the small singularity near $\mathrm{Rh}_{0.30} \mathrm{Pd}_{0.70}$ is due to the top of the fourth band. The stippled parts of the curves have not been directly calculated, but have been estimated as explained in the text and in Table $\mathrm{V}$.

surements on PdRh alloys ${ }^{51}$; but this goes beyond the scope of the present paper.

The derived values of $\gamma$ and $\chi$ enhancements for Pd are in excellent agreement with the values: $m^{*} / m=1.73$ and $D=10$ obtained by Chouteau et al..$^{52}$ These authors measured the concentration dependence of $\gamma$ and $\chi$ for dilute PdNi alloys and interpreted their results according to the local exchange-enhancement theory of Lederer and Mills. ${ }^{53}$

\section{B. dHvA Data}

Until now the most detailed and accurate experimental information on the Fermi-surface properties of the fcc transition metals has been obtained by the dHvA technique. Comparison between the measured and theoretical extremal areas provides a most necessary check of the theoretical band structure, and the ratio between measured and theoretical cyclotron masses is believed to yield a good estimate of the enhancement factors due to many-body interactions.

In order to define a convenient measure for the agreement between experimental and theoretical extremal areas we note that if hybridization effects are neglected, a plausible first correction to a theoretical Fermi surface is a small rigid shift of each band relative to the Fermi level, such as to fit the dHvA areas: 
TABLE VI. Total number of states $n$ and density of states $N$ for Pd (Fig. 12). The dots indicate the computed volumes of constant-energy surfaces, and the curves are given by the fit $n=n(0)+N(0) \Delta E+a \Delta E^{3 / 2}$ $+b \Delta E^{2}, N=N(0)+\frac{3}{2} a \Delta E^{1 / 2}+2 b \Delta E$, where $\Delta E^{3 / 2} \equiv 0$ and $\Delta E^{1 / 2} \equiv 0$ for $\Delta E \leqslant 0$, further $\Delta E \equiv E-E\left(P_{1}\right)$ and $\Delta E_{F}$ $=1.89$. These expressions are valid in the range -1 $\lesssim \Delta E \lesssim 8$. Energies are in $\mathrm{mRy}$ and number of states in $10^{-3}$ states/atom. With $N$ in (states/atom)/Ry the electronic specific-heat coefficient and the Pauli susceptibility are, respectively, $\gamma_{\text {band }}=0.1735 \mathrm{~N} \mathrm{~mJ} / \mathrm{mole}^{\circ} \mathrm{K}^{2}$, $\chi_{P}=2.377 \times 10^{-6} \mathrm{Nemu} / \mathrm{mole}$.

\begin{tabular}{crccc}
\hline \hline Band & $n(0)$ & $N(0)$ & $a$ & $b$ \\
\hline 6 & 367.0 & 3.40 & 0.0 & 0.0 \\
5 & -425.3 & 33.82 & -1.486 & -0.590 \\
$4+5+6$ & -66.3 & 38.06 & -1.458 & -0.615 \\
\hline \hline
\end{tabular}

$$
\begin{aligned}
\delta_{i} & \equiv \delta\left(E_{F}-E_{i}\right)=\left\langle\frac{1}{\pi} \frac{A^{\text {expt }}-A^{\text {theor }}}{m^{\text {theor }} / m_{0}}\right\rangle_{i} \\
& \equiv \frac{n_{i}^{\text {expt }}-n_{i}^{\text {theor }}}{N_{i}^{\text {theor }}} .
\end{aligned}
$$

$E_{i}$ is a characteristic energy of the $i$ th band, and the bracket indicates a mean value of different orbits over the $i$ th sheet. Conservation of particles yields the constraint

$$
\sum_{i} N_{i}^{\text {theor }} \delta\left(E_{F}-E_{i}\right)=0,
$$

which determines $\delta E_{F}$ and expresses the previously mentioned "clamping" of the Fermi level by a band with a high density of states.

Since masses of small Fermi-surface sheets tend to be small, we generally expect larger relative

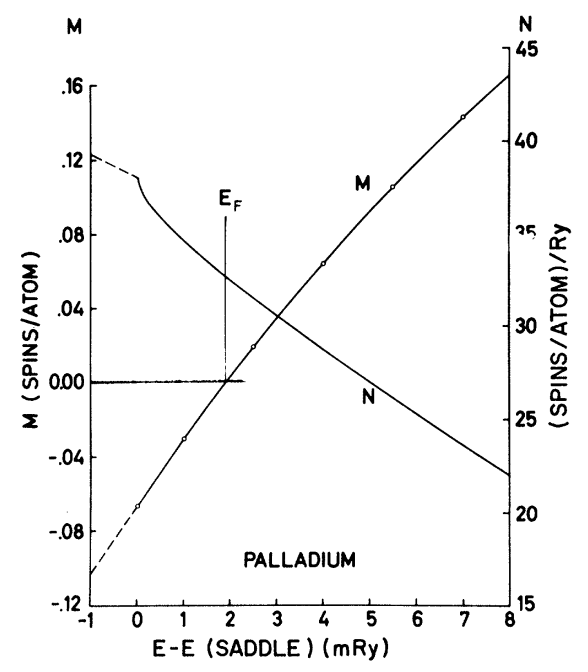

FIG. 12. Detailed density of states $N$ and the number of states $M$ in excess of 10 states/atom for Pd (see further Table VI).
TABLE VII. Experimental and theoretical values of the electronic specific-heat coefficient.

\begin{tabular}{lcccc}
\hline & $\mathrm{Rh}$ & $\mathrm{Ir}$ & $\mathrm{Pd}$ & $\mathrm{Pt}$ \\
\hline $\begin{array}{l}\text { Experimental } \\
\left(\mathrm{mJ} / \text { mole }^{\circ} \mathrm{K}^{2}\right)\end{array}$ & $4.65 \pm 0.02$ & 3.27 & $9.42 \pm 0.02$ & $6.56 \pm 0.03$ \\
$\begin{array}{l}\text { Theoretical } \gamma \\
\left(\mathrm{mJ} / \text { mole }^{\circ} \mathrm{K}^{2}\right)\end{array}$ & 3.24 & 2.39 & 5.67 & 4.02 \\
$\begin{array}{l}\text { Enhancement } \\
\text { factor }\end{array}$ & 1.44 & 1.37 & 1.66 & 1.63 \\
\hline \hline
\end{tabular}

discrepancies between experimental and theoretical areas for smaller than for larger sheets; the energy shifts $\delta$, however, are of the same order of magnitude. Moreover, the theoretical masses of the smaller sheets are quite reliable since small sheets arise near band extrema, where the bands are parabolic and band masses accordingly independent of energy.

We are mainly interested in those parts of the Fermi surface which contribute most to the total density of states, i.e., large highly $d$-like sheets. Orbits associated with such sheets have large cyclotron masses, and unfortunately, dHvA amplitudes therefrom can hardly be detected in the presently obtainable temperature range, even for the purest specimens. At present, data on the fifth band orbits are scarce, but the situation may soon change. However, from extensive dHvA data on all other sheets $\delta\left(E_{F}-E_{5}\right)$ may be found, and most importantly, from the dHvA data on all other sheets plus

Concentration of $\mathrm{Rh}$ or $\mathrm{Ag}$ in $\mathrm{Pd}$

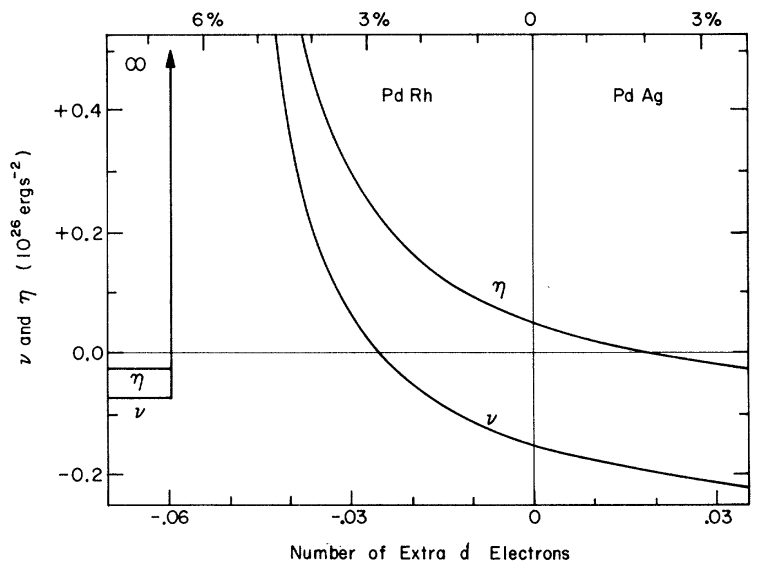

FIG. 13. Band-structure parameters $\eta$ and $\nu$ entering the second-order terms of the $d$-spin susceptibility as a function of temperature and magnetic field, respectively [Eqs. (2) - (6)]. The parameters have been derived from the Pd density of states (Table VI), and the rigid-band model has been employed. 
a reliable value of the experimental electron specific-heat coefficient, the average mass enhancement on the fifth sheet may be extracted.

\section{Rhodium}

The central extremal areas in the symmetry directions and the corresponding masses have been calculated and are compared in Ref. 6 with the experimental results of Coleridge ${ }^{54}(X 3, X 4$, and $L 4)$ and of Ketterson et al. ${ }^{55}$ ( $\Gamma 5$ and $\Gamma 6$ ). The agreement between theoretical and experimental areas is very good, the $\delta_{i}$ 's being less than $4 \mathrm{mRy}$.

Exceptions are the only two measured orbits for $X 4$ and $\Gamma 5$. The $X 4$ area was difficult to measure, ${ }^{54}$ so its accuracy is presumably low. The energy shift calculated from the areas and the theoretical mass of the $\Gamma 5$ orbit is $\delta_{5}=-7.7 \mathrm{mRy}$, whereas the number obtained from all other bands ${ }^{56}$ is -1.5 mRy. We therefore believe that the experimental (110) area of $\Gamma 5$ belongs to an extremal noncentral orbit (which we have not attempted to calculate).

The many-body enhancement factors seem to be about 1.1 for all cyclotron masses belonging to the small hole pockets of the third and fourth bands, but only a minute part of the total number of states is included in these (Table I), and the total enhancement is 1.44 (Table VII). Cyclotron masses of $\Gamma 6$ may soon be available, ${ }^{55}$ so that the most interesting enhancement factors of $\Gamma 6$ and $\Gamma 5$ may be found.

\section{Iridium}

The only available Ir data are recent preliminary results for the smallest hole pockets $X 3$ by Dixon and Grodski. ${ }^{9}$ The agreement with our theoretical areas is relatively poor, $\delta_{3} \sim 12 \mathrm{mRy}$, compared to the excellent agreement obtained for $\mathrm{Rh}, \mathrm{Pd}$, and Pt. If the experimental values are correct, the theoretical $s p-d$ band separation is inaccurate, but whether the $s p$ band sits too high or too low is hard to say since hybridization is important: If the $s p$ band is lowered, the $X 3$ becomes more $s p$-like and it shrinks; if the $s p$ band is raised, the Fermi level will, to a minor extent, be dragged with it, and the $X 3$ probably shrinks again. Detailed measurements of the Fermi velocities of $X 3$, by inversion of the cyclotron masses, and an experimental statement concerning the existence of an $L 4$ pocket for Ir could clarify the situation. The mass enhancement for $X 3$ seems to be 1.1, as was the case in Rh. \{Note added in manuscript. Most recent data for the $X 4$ pocket are in agreement with our prediction, $\delta_{4} \sim-3 \mathrm{mRy}$, but the $X 3$ data confirm those of Dixon and Grodski. [S. Hörnfeldt, Solid State Commun 8, 673 (1970)]\}.

\section{Palladium}

The recent areas and masses of Windmiller,
Ketterson, and Hörnfeldt ${ }^{10}$ have been compared with the theoretical values in Ref. 10, and the agreement between areas is amazingly good, corresponding to $\delta$ values in all cases of $3 \mathrm{mRy}$ or less.

The $\Gamma 6$ sheet is closed and has inversion symmetry, its radius vector $r$ is a single-valued function of $\theta$ and $\varphi$ and therefore the experimental central areas $A(\theta, \varphi)$ may be uniquely transformed into $r(\theta, \varphi) .{ }^{57}$ This inversion was first applied ${ }^{58}$ to the dHvA areas of Vuillemin and Priestly, ${ }^{59}$ but with the recent more extensive $A(\theta, \varphi)$ data $^{10}$ much better accuracy has been achieved. ${ }^{60}$ The latter experimental $\Gamma 6$ radii have a maximum deviation of no more than $2 \%$ from our theoretical values; this worst case is along $\Lambda$ (Fig. 5), where the theoretical radius is the larger.

Unfortunately, the experimental information on $X W 5$ is meager; only the small low-mass orbit $\alpha$, which is the nearly circular (100) orbit centered at $W$ (Fig. 6) has been measured; the masses of all other extremal orbits on $X W 5$ are very high. As estimated from the $\alpha$ orbit, the $\delta_{5}$ shift is about $-0.9 \mathrm{mRy}$. The other energy shifts are $\delta_{6} \sim 2 \mathrm{mRy}$ and $\delta_{4} \sim 1.2 \mathrm{mRy}$ thus yielding $\delta_{5} \sim 0.3 \mathrm{mRy}$, which is in reasonable agreement with the above.

The average mass enhancements on $\Gamma 6$ and $X 4$ are 1.52 and 1.54 , respectively, and with a specific-heat enhancement of 1.66 , the average enhancement on $X W 5$ must be about 1.68 , which is in fair agreement with the mass enhancement of the $\alpha$ orbit.

\section{Platinum}

As shown in Ref. 6 the agreement between the areas of Ketterson and Windmiller ${ }^{61}$ and the theoretical values is again surprisingly good, corresponding to $\delta$ values in all cases of $5 \mathrm{mRy}$ or less.

The areas of $\Gamma 6$ have been measured in detail and inverted into radii by Ketterson et al. ${ }^{62}$; this work also contains the comparison with our theoretical radii. As for $\mathrm{Pd}$ the maximum deviation is less than $2 \%$, and a rigid shift $\delta_{6}$ of about $4.2 \mathrm{mRy}$ would bring the maximum deviation of the radii down below $1 \%$, but it cannot be reduced further by this means, since $\Gamma 6$ is severely $d$ hybridized.

For Pt the high-mass orbits $\gamma$ and $\delta$ on the important $X W 5$ sheet have been measured. These (110) orbits are noncentral, and it is satisfying that they have been found also in the theoretical model (Fig. 14), and that the areas of these odd-shaped orbits agree so well. The heavy (110) orbit $\beta$ is centered at $X$ and passes the tip of four fins; it has not been detected yet. From the data on the $\alpha$, $\gamma$, and $\delta$ orbits $\delta_{5}$ is about $-1 \mathrm{mRy} ; \delta_{6}$ and $\delta_{4}$ are 4 and $-3 \mathrm{mRy}$, respectively, and these three numbers are consistent, even in this case of rather 


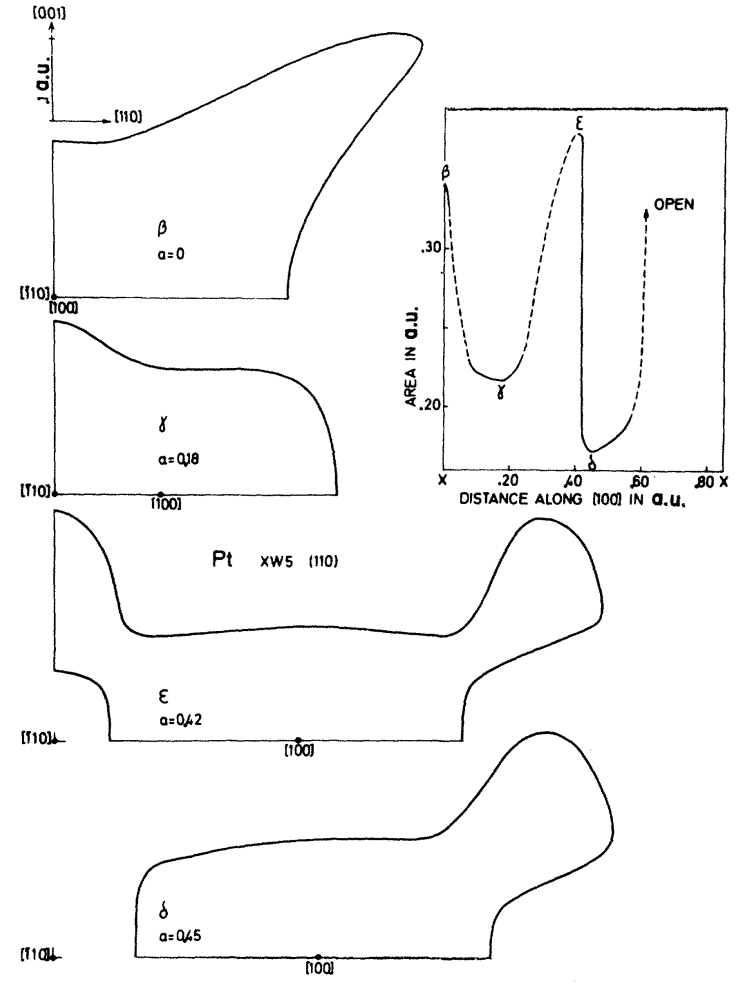

FIG. 14. Extremal (110) areas of the Pt XW5 (Fig. 6). These cross sections are at $45^{\circ}$ to the "cylinder" direction [110], the $\beta$ orbit is centered at the symmetry point $X$, and the distance $a$ is measured along [100] in atomic units. The $\epsilon$ orbit shown is nearly extremal, and results when the cross sections of two intersecting cylinders separate. In the search for extremal areas the full-line portions of the area versus distance plot have been calculated. Comparisons with experiment are given in Ref. 6 .

severe hybridization.

Not only the experimental central areas, but also the cyclotron masses, $m(\theta, \varphi)$, of $\Gamma 6$ have been inverted. $^{62}$ These experimental Fermi velocities have been compared with our theoretical values (Fig. 7) in Ref. 62, and it is striking that the same distinct variation of $\vec{v}$ versus $\theta$ and $\varphi$ is found in both experimental and theoretical results, so that the many-body renormalization factor is nearly isotropic. This is even more convincing, when it is realized that, although the $\mathrm{Pd}$ and $\mathrm{Pt} \Gamma 6$ radii are very similar (Fig. 5), the theoretical velocities are extremely different (Fig. 7). The experimental Pd velocities will probably soon be available, but preliminary results ${ }^{60}$ indicate that their anisotropy is similar to our findings for Pd and in this respect distinct from the Pt values. These same results may be found from the cyclotron masses: For the hybridized $\Gamma 6$ of $\mathrm{Pt}$ the masses are anisotropic, whereas the mass enhancements are nearly isotropic, and these $\Gamma 6$ mass enhancements are nearly equal for $\mathrm{Pd}$ and $\mathrm{Pt}$.

Integration of the experimental $\Gamma 6$ velocities yielded a partial density of states of 6.35 states/ atom/Ry and hence an average enhancement of 1.50 . With a total enhancement of 1.63 (Table VII) the average enhancement on $X W 5$ must be 1.67 , which is consistent with the enhancements on the $\alpha$ and $\gamma$ orbits. The theoretical $\delta$ mass may be too large due to overestimation of the circumference of this orbit.

The most significant results on the mass enhancements may be summarized as follows: The average mass enhancement on $X W 5$ is the same for Pt and Pd (1.67-1.68), and so is the mass enhancement on $\Gamma 6(1.50-1.52)$. The $X W 5$ enhancement is significantly larger than the $\Gamma 6$ enhancement. For Pd there is a great difference between, the $\Gamma 6$ and $X W 5$ partial state densities; for $\mathrm{Pt}$ severe hybridization occurs, and the density-ofstates difference is smaller. This explains why the total enhancement of $\mathrm{Pd}$ is slightly larger than that of $\mathrm{Pt}$.

\section{DISCUSSION}

The important question concerning the significance of the derived many-body renormalization factors is: How meaningful are our band velocities, masses, and density of states? We cannot answer this question, but we shall give a qualitative justification of our approach.

There are three factors which essentially determine the nonrelativistic band structure of a transition metal ${ }^{7}$ : the lattice structure, the position of the $d$ resonance with respect to the bottom of the $s p$ band, and the width of this resonance. Since the agreement with the experimental Fermi-surface dimensions is excellent, we believe that the structural parts of the bands are correct, i.e., a sufficient number of reciprocal-lattice vectors were included in the RAPW expansions. A less trivial consequence of this excellent agreement is that the position of the $d$ band with respect to the $s p$ band is probably correct within a shift of no more than a few times $\delta\left(E_{5}-E_{6}\right) \sim 5 \mathrm{mRy}$. But at present we do not really have any independent check of the resonance width $W$, which determines the hybridization strength and the scale of the $d$ band. ${ }^{7}$ Whereas for fixed structure constants and position of the resonance the theoretical Fermi surface usually depends only weakly on $W$, namely, via the hybridization interaction, the velocities, masses, and density of states are directly or inversely proportional to $W$. For Pt the agreement between the theoretical and experimental anisotropy of velocities on the severely hybridized $\Gamma 6$ sheet might indicate that $W$ has a 
reasonable value, but generally we must base our belief, that agreement for the Fermi surface ensures correct one-electron values for band velocities, masses, and density of states, on an interdependence between the width and the position of the resonance. Such an interdependence is plausible for the kind of local potential we have used to simulate the effects of exchange and correlations. ${ }^{63}$

Let us assume that the potential has the muffintin form, ${ }^{64}$ so that the Schrödinger equation separates, and the radial equation contains the centrifugal term $l(l+1) / r^{2}$. In Fig. 15 we show the atomic and muffin-tin potentials used for Pt plus their respective effective $d$ potentials, which include the centrifugal term. It may be seen how the construction of the muffin-tin potential from overlapping atomic potentials gives rise to a barrier in the effective $d$ potential and thereby to the resonance. The absolute position of the $d$ resonance depends on the potential inside the core, which does not differ greatly from the atomic potential; accordingly, the atomic $5 d$ level and the $d$ resonance of the muffintin potential were found to coincide within $20 \mathrm{mRy}$. The bottom of the $s p$ band, however, depends on the outer parts of the muffin-tin potential, and its position is usually not far from the MT0. ${ }^{14}$ The width of the resonance is determined by the potential barrier, which again depends critically on the outer parts of the potential. Therefore, both the width of the resonance and its position within the $s p$ band are extremely sensitive to details in the

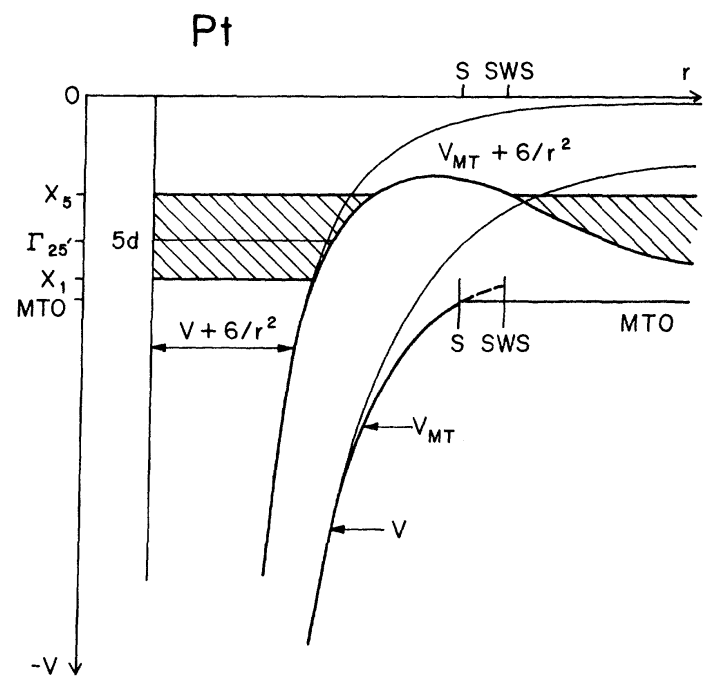

FIG. 15. $5 d$ energies and potential terms of the radial equation for $\mathrm{Pt}$. Weak lines apply to the atomic potential and solid lines to the muffin-tin potential. $6 / r^{2}$ is the centrifugal term for $d$ states. $S$ and $S W S$ are the radii of muffin-tin and Wigner-Seitz spheres, respectively. outer parts of the muffin-tin potential. ${ }^{64}$ These remarks are due to Ziman. ${ }^{14}$ Our point is that it seems impossible within the local-potential approximation to perturb the potential in any physically reasonable way, such that the width of the resonance is significantly changed at the same time as its relative position remains constant. For instance, the Pt potential derived from a $5 d^{10}$ rather than from a $5 d^{9} 6 s$ configuration is weaker, where the concentration of $5 d$ electrons is high, but the MT0 is almost the same as for the $5 d^{9} 6 s$ potential; thus, the $d$ band will be broader and will sit higher with respect to the $s p$ band. The same trend will be followed if the amount of Slater exchange is reduced: The exchange potential is negative and proportional to the cube root of the charge density so exchange reduction will make the inner parts of the potential weaker. By actually calculating part of the band structure for Pt and Ir with $\frac{2}{3}$ reduced Slater exchange, we found that the widening of the $d$ band amounted to about $20 \%$, and at the same time the $d$ band was raised about $130 \mathrm{mRy}$. By plotting position versus width of the $d$ band from a large number of independent nonrelativistic energy-band calculations for fcc transition metals Connolly ${ }^{11}$ found that the results tended to lie on a straight line of slope $\approx 2$. It therefore seems that together with any significant change, say, $10 \%$, in the $d$ bandwidth for $\mathrm{Rh}, \mathrm{Pd}$, Ir, or Pt there would be a shift in the $s p-d$ position of the order $100 \mathrm{mRy}$; and this far exceeds our estimated $s p$ - $d$ shift.

Our calculations were not carried to self-consistency, therefore the fact that we found good agreement with dHvA experiments using the full Slater exchange does not contradict a recent view ${ }^{65}$ that the exchange should be reduced by a factor close to 0. 7; moreover, self-consistent calculations for $\mathrm{Ni}^{11}$ and $\mathrm{Cu}{ }^{66}$ have shown that the final band structures obtained with an exchange factor of 0.67 were close to initial, not self-consistent results which made use of full Slater exchange. However, this result depends to some extent on the starting potential (choice of configuration), and our claim is merely that having obtained good agreement with dHvA areas by using some physically reasonable potential, the derived slopes of these bands at the Fermi energy are reasonable.

When relativistic effects are included, in order for the above discussion to be valid, it is important that, when the mass-velocity and Darwin terms are large such as for Ir and Pt, they should be calculated correctly, because these terms are effective in lowering the $s p$ band (and slightly narrowing the $d$ band, Sec. IIIA). Also the spin-orbit coupling must be correctly dealt with, since it changes the upper $d$ bands substantially. Let us try to get an idea of how much the density of states at the Fermi 
level is influenced by the relativistic terms. There are essentially two effects: The distortion of the $d$ band brought about mainly by the spin-orbit coupling, and the relocation of the Fermi level due to the shift in $s p-d$ band position brought about by the mass-velocity and Darwin terms. Because of hybridization, however, the first effect depends very much on the $d$-band position, e.g., removal of spin-orbit coupling from our relativistic Pt bands, where the $p$-like $L_{6}^{-}$level falls between the spinorbit split $d$-like $L_{4}^{+} L_{5}^{+}$and $L_{6}^{+}$levels, has a quite different effect than has the removal of spinorbit coupling from the Pd bands, where at $L$ the $p$ level lies well above the top of the $d$ band. Let us therefore first switch off the spin-orbit coupling, then the shift in $d$-band position caused by the mass-velocity and Darwin terms, and finally try to readjust the Fermi level.

Several authors ${ }^{8,25}$ have found that, for the nonrelativistic energy bands of fcc transition metals, the high peak in the density of states at the top of the $d$ band is not affected by changes in the position of the $s p$ band; this is because the states contributing to this peak are purely $d$ like. A characteristic value of the density of states at an energy fixed within the $d$ band is the peak value. Five different energy bands for $\mathrm{Cu}$ and $\mathrm{Ni}^{8,25,26,67}$ with $d$-band positions at $L$ ranging from $L_{3}$ being $100 \mathrm{mRy}$ below the $p$-like $L_{2}^{\prime}$ to $L_{3}$ being $70 \mathrm{mRy}$ above $L_{2}^{\prime}$ and with correspondingly varying $d$ bandwidths, all yield 24 states/atom for the normalized density of states at the peak, i.e., density of states times $d$ bandwidth. We take this as the nonrelativistic value.

For Pd we may now visualize the effect of spinorbit coupling by considering the details of the uppermost $d$ band around $L$ (Fig. 10): When spinorbit coupling is removed the fifth and fourth bands are degenerate at $L_{3}$ and $X_{5}$, so the fifth band moves roughly $10 \mathrm{mRy}$ downwards, and at the same time it flattens, especially near $L$. The $\overrightarrow{\mathrm{k}}$ space location of the $P_{2}$ saddle point remains constant, ${ }^{18}$ but $P_{1}$ appears at $L$. Consequently, we expect that removal of spin-orbit coupling increases the peak value of the density of states; we find for $\mathrm{Pd} \mathrm{a}$ normalized peak value of 19 states/atom, so the increase is $25 \%$. Removal of the mass-velocity and Darwin terms may be estimated to yield an upwards shift of $s p$-band position, which near the top of the $d$ band, is of the order of $5 \mathrm{mRy}$; so the total shift between the $s p$ band and the uppermost $d$ band is about $15 \mathrm{mRy}$. The Fermi level is thereby lifted about $1.5 \mathrm{mRy}$ relative to the fifth band [Eq. (8)] and accordingly, the density of states at the Fermi level is reduced by approximately $10 \%$. For Pd our estimate of the total effect of the relativistic terms on the density of states at the Fermi level is therefore a $10-20 \%$ decrease.

For Pt we have not investigated the peak in the density of states, but from the bands of Fig. 1 and the study of Hodges et al. ${ }^{25}$ we may get an idea of the spin-orbit effects. By means of their interpolation scheme Hodges et al. added spin-orbit coupling in the tight-binding approximation to the energy bands of $\mathrm{Ni}$, and since for these bands the $p$-like $L_{2}^{\prime}$ level is just of the order of $10 \mathrm{mRy}$ above the $d$ like $L_{3}$, this study seems applicable to Pt (but not to $\mathrm{Pd}$ ). The highest value of the spin-orbit parameter $\xi_{d}$ was $61 \mathrm{mRy}$, which almost equals our value of $\xi_{d}$ at the Fermi level (Fig. 3). As previously remarked, in the spin-orbit decoupled case, the saddle points of the fifth band are the $P_{2}$ near the midpoint of $Q$ and the $P_{1}$ at $L$. With the $s p$-band position appropriate for relativistic Pt, the Fermi level is slightly above $E\left(P_{1}\right)$. If we now switch on the spin-orbit coupling, the $L_{3}$ level belonging to the fourth and fifth bands splits, and its uppermost level $L_{4}^{+} L_{5}^{+}$inverts with the $L_{6}^{-}\left(L_{2}^{\prime}\right)$ level; consequently, the fifth band becomes $p$-like at $L$, and exhibits a saddlepoint of type $P_{2}$. The $P_{1}$ saddlepoint moves from $L$ along $Q$, and for the full strength of the coupling this saddlepoint lies at about the same distance from $L$ as the corresponding $P_{1}$ saddlepoint for $\mathrm{Pd}$ at $[L X]$. For $\mathrm{Pt}$ the density-of-states peak has two singularities on the low-energy side: $P_{2}$ at $L$ and the purely $d$-like $P_{2}$ on $Q$ midway between $L$ and $W$, which also existed for $\mathrm{Pd}$ and $\mathrm{Rh}$. On the high-energy side there is the $P_{1}$ singularity. For this complicated case, where spin-orbit coupling and $s p-d$ hybridization are effective at the same time, Hodges et al. found that the normalized density of states at the peak was reduced from 24 to about 22 states/atom, but at the same time the peak became more narrow, and the Fermi level moved upwards, away from high-density parts of the peak. Accordingly, the density of states at the Fermi level was found to be about $40 \%$ higher in the decoupled case than for $\xi_{d}=61 \mathrm{mRy}$. Since, for this value of the spin-orbit parameter, the tight-binding result of Hodges et $a l$. yielded a spin-orbit splitting at $L$ which was only about $\frac{2}{3}$ of our RAPW value, the effect of spinorbit coupling in the present RAPW calculation may be even larger. Near the top of the $d$ band the mass-velocity and Darwin terms lower the $s p$ band with respect to the $d$ band by approximately $20 \mathrm{mRy}$ (Sec. IIIA), but the extent to which the Fermi level is dragged along with the $s p$ band, and the spin-orbit effect thereby compensated, cannot easily be estimated because of the severe hybridization.

We have seen that the difference in strength of the relativistic terms between $\mathrm{Pd}$ and $\mathrm{Pt}$ has a significant effect on both the shape of the density of states and on the position of the Fermi level; however, 
for the value of the density of states at the Fermi level these two effects tend to cancel. This could explain our notable observation (Table III) that going from $\mathrm{Rh}$ to Ir or from $\mathrm{Pd}$ to $\mathrm{Pt}$, in both cases the density of states at the Fermi level scales according to $d$ bandwidths, within $3 \%$.

\section{CONCLUSION}

As all four transition metals have the fcc structure, the shape of the energy bands looks very similar and so do the Fermi surfaces of isoelectronic metals. But there are significant differences in width and position of the $d$ band, hybridization between $s p$ and $d$ bands, and spin-orbit effects. For isoelectronic metals the total state densities at the Fermi levels scale almost exactly as the inverse of the $d$ bandwidths (Table III), however this probably does not mean that $d$-band positions and spin-orbit couplings have no effect, but rather that these effects tend to cancel out. The partial state densities of the individual sheets of Fermi surface, the cyclotron masses and the group velocities at the Fermi surface do not scale according to $d$ bandwidths among isoelectronic metals, but depend significantly upon $d$-band position and spinorbit coupling. Pd has the least hybridized Fermi surface; $\Gamma 6$ has much $s p$ character, and all other sheets are almost purely $d$-like. The Ir Fermi surface is most severely hybridized. For these four metals the fifth band is particularly important, it contributes nearly $90 \%$ to the total state density of $\mathrm{Pd}$ and over half the state density of Ir. Moreover, the closed $\Gamma 5$ sheet of $\mathrm{Rh}$ and $\mathrm{Ir}$ is of different topology than the open $X W 5$ sheet of $\mathrm{Pd}$ and $\mathrm{Pt}$, and this topological transition gives rise to the high density-of-states peak at the top of the $d$ band between the Fermi levels of the 9- and 10-electron metals.

The theoretical Fermi surfaces are in excellent agreement with the results of recent accurate and detailed dHvA measurements, but unfortunately, experimental data on the sheets of the fifth band are still meager. However, since the theoretical Fermi level was found by the requirement that the sum of volumes for the different sheets should yield the correct number of states, the agreement between theory and experiments for all but one sheet of Fermi surface implies agreement in the volume also for this one sheet. Except for Ir, where the experimental data are preliminary, the differences between experimental and theoretical Fermi surfaces mainly correspond to shifts in the positions of the theoretical bands of $5 \mathrm{mRy}$ or less. In view of this surprisingly good agreement, the theoretical state densities of Tables I and V-VII seem meaningful. The derived enhancement factors for the electron specific-heat coefficients (Table VII) seem mainly to reflect the electron-phonon enhancements, and the value for Ir is in close agreement with the theoretical estimate for the electron-phonon enhancement of McMillan, ${ }^{37}$ but the larger values for $\mathrm{Pt}$ and Pd may indicate a contribution from spin fluctuations. Taking the experimental cyclotron masses for Pt and Pd into account, we also find the partial enhancements of the $\Gamma 6$ sheets and the highly $d$-like $X W 5$ sheets. The numbers are 1.51 and 1.68 for $\Gamma 6$ and $X W 5$, respectively both for $\mathrm{Pt}$ and $\mathrm{Pd}$. Here again the higher enhancement of $d$-electron masses might be due to the interaction with spin fluctuations. Comparison with the electronic specific heat of PdRh and dilute PdAg alloys indicates that the rigid-band approximation does not hold very well. The theoretical peak in the state density falls about $7 \mathrm{mRy}$ below the Fermi level of $\mathrm{Pd}$, which corresponds to a $\mathrm{Pd}_{0.80} \mathrm{Rh}_{0.20}$ alloy, and this is consistent with photoemission and optical experiments, but the peak value of the electronic specific-heat coefficient and of the susceptibility is found near the $\mathrm{Pd}_{0.95} \mathrm{Rh}_{0.05}$ alloy, i.e., $2 \mathrm{mRy}$ below the Pd Fermi level according to the theoretical state density for Pd. The susceptibility enhancement is about 10 and 4 for $\mathrm{Pd}$ and $\mathrm{Pt}$, respectively, and a van Hove singularity just about $2 \mathrm{mRy}$ below the Fermi level of Pd seems to explain the peculiar field and temperature dependence of the susceptibility for Pd and dilute alloys.

\section{ACKNOWLEDGMENTS}

The author wishes to express sincere gratitude to Professor A. R. Mackintosh, who proposed this research and supervised it with warm interest. Moreover, through the courtesy of Professor Mackintosh, many results of his Pt calculation are published here for the first time. The basic RAPW programs used in this calculation were kindly supplied by Professor T. L. Loucks. Valuable discussions with Professor J. R. Schrieffer, Dr. J. B. Ketterson, Dr. L. R. Windmiller, and Dr. F. M. Mueller are also gratefully acknowledged. We have benefited from the use of the facilities of the Northern Europe University Computing Center, Lyngby, Denmark, where most of the calculations were performed.

\section{APPENDIX A: RELATIVISTIC EFFECTS IN THE APW METHOD}

With the APW and RAPW methods, respectively, the Schrödinger and Dirac equations may be approximately solved in a periodic muffin-tin potential. ${ }^{5}$ When comparing results, however, the relativistic effects are usually interpreted in the language of the Pauli equation, i. e., as the sum of massvelocity, Darwin, and spin-orbit terms. In the original expression for the Dirac RAPW matrix ${ }^{21}$ 
the Pauli and Schrödinger limits were not transparent, but after various comments ${ }^{19,68-70}$ it may easily be shown that, with the applied approximations, ${ }^{70}$ the APW matrix has one and the same form whether Schrödinger, Pauli, or Dirac Hamiltonians are employed, namely,

$$
\begin{aligned}
\left\langle\overrightarrow{\mathrm{K}}_{i}, m_{i}|H-E| \overrightarrow{\mathrm{K}}_{j}, m_{j}\right\rangle & \\
= & \left.\overrightarrow{\mathrm{K}}_{i} \cdot \overrightarrow{\mathrm{K}}_{j}-E\right) O\left(\left|\overrightarrow{\mathrm{K}}_{i}-\overrightarrow{\mathrm{K}}_{j}\right|\right)\left\langle m_{i} \mid m_{j}\right\rangle \\
& +\frac{4 \pi S^{2}}{\Omega} \sum_{l=0}^{\infty} P_{l}(i j) j_{l}(i) j_{l}(j) \alpha_{l}(E)\left\langle m_{i} \mid m_{j}\right\rangle \\
& +\frac{4 \pi S^{2}}{\Omega} \sum_{i=1}^{\infty} P_{l}^{\prime}(i j) j_{l}(i) j_{l}(j) \beta_{l}(E) \\
& \times\left\langle m_{i}\left|i \vec{\sigma} \cdot\left(\hat{K}_{j} \times \hat{K}_{i}\right)\right| m_{j}\right\rangle .
\end{aligned}
$$

Here, $\overrightarrow{\mathrm{K}}_{i}=\overrightarrow{\mathrm{k}}+\overrightarrow{\mathrm{g}}_{i}$, and $m_{i}$ indicates the spin direction of the plane-wave part of the APW. $O(K)$ is the Fourier transform of a step function, being zero inside the MTS and unity outside. $S$ is the muffin-tin radius, $\Omega$ is the volume of the unit cell, $P$ is a Legendre polynomial, and $j_{l}$ is a spherical Bessel function. ( $i)$ and $(i j)$ stand for $\left(K_{i} S\right)$ and $\left(\hat{K}_{i} \cdot \hat{K}_{j}\right)$, respectively. $\vec{\sigma}$ is the Pauli matrix, and we have used atomic units. Moreover,

Schrödinger equation:

$$
\alpha_{l}(E)=(2 l+1)\left(R^{\prime} / R\right)_{l} ;
$$

Pauli equation:

$$
\alpha_{l}(E)=(l+1)\left(R^{\prime} / R\right)_{-l-1}+l\left(R^{\prime} / R\right)_{l} ;
$$

Dirac equation:

$$
\alpha_{l}(E)=(l+1)(c f / g)_{-l-1}+l(c f / g)_{l} ;
$$

and

Schrödinger equation:

$$
\beta_{l}(E)=0
$$

Pauli equation:

$$
\beta_{l}(E)=\left(R^{\prime} / R\right)_{-l-1}-\left(R^{\prime} / R\right)_{l} ;
$$

Dirac equation:

$$
\beta_{l}(E)=(c f / g)_{-l-1}-(c f / g)_{l}+(2 l+1) / S .
$$

$R_{l}(E, r), R_{k}(E, r), f_{k}(E, r)$, and $g_{k}(E, r)$ are the solutions of the radial Schrödinger, Pauli, or Dirac equations, where the quantum number $\kappa$ is the eigenvalue of $-(\vec{\sigma} \cdot \vec{I}+1)$. In the above expressions for $\alpha$ and $\beta$ the radial functions are evaluated at the radius of the MTS, and here the radial Dirac equations yield

$$
(c f / g)_{\kappa} \approx\left(g^{\prime} / g\right)_{\kappa}+(\kappa+1) / S,
$$

which makes the passage to the Pauli expressions clear. In Fig. 16 we show the Dirac logarithmic derivatives for $\mathrm{Pt}$.
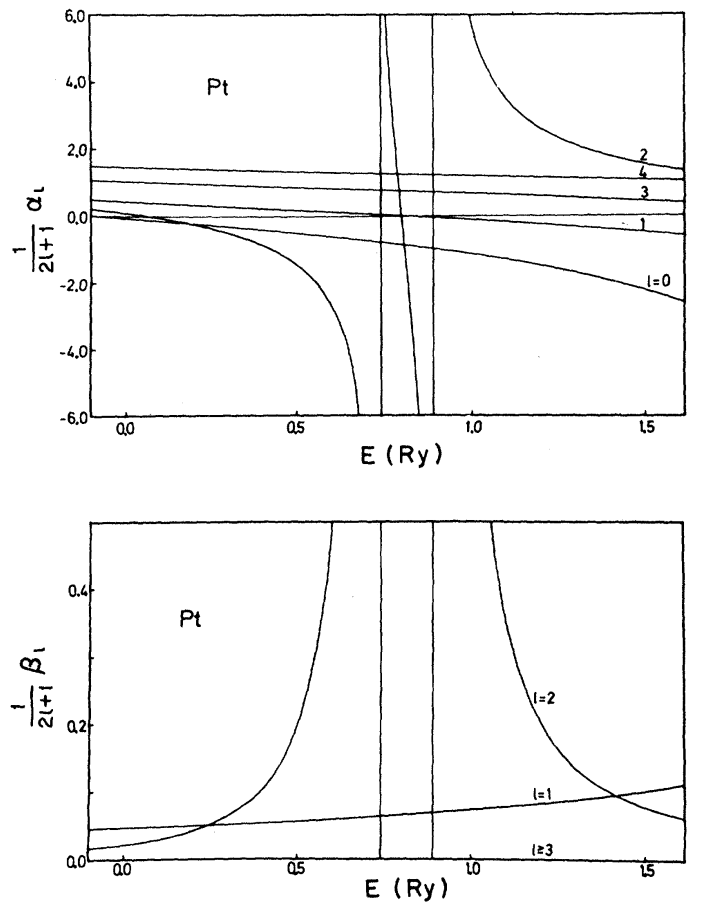

FIG. 16. "Dirac logarthmic derivatives" of Pt.

When the small components $f_{k}(E, r)$ are eliminated from the radial Dirac equations and all terms of order higher than $c^{-2}$ are neglected, we arrive at the radial Pauli equation:

$$
\begin{aligned}
& {\left[-d^{2} / d r^{2}+l(l+1) / r^{2}+V(r)-E\right] r R_{\kappa}} \\
& \quad=c^{-2}\left[(V-E)^{2}-\left(V^{\prime \prime} / 2+V^{\prime} / r\right)+V^{\prime}(1+\kappa) / r\right] r R_{\kappa},
\end{aligned}
$$

where we have written $R_{k}$ instead of $g_{k}$. The relativistic terms on the right-hand side are the massvelocity, Darwin, and spin-orbit terms, respectively.

So due to the requirement that inside the MTS an APW should be a solution of the appropriate Hamiltonain, i.e., Dirac, Pauli, or Schrödinger, and due to the neglect of relativistic terms outside the $\mathrm{MTS}^{5,70}$ the APW matrix [Eq. (A1)] takes any selection of relativistic terms into account, when the coefficients $\alpha$ and $\beta$ are derived from the appropriate radial equation.

\section{APPENDIX B: SPIN-ORBIT COUPLING}

Because $\beta$ vanishes, when spin-orbit coupling is neglected [Eqs. (A2e) and (A4)], and due to the form of the third term of the APW matrix [Eq. (A1)], this term has been called the spin-orbit term. ${ }^{19,70}$ Accordingly, in order to remove spin-orbit coupling from a relativistic calculation, this term has 
been neglected, by setting $\beta=0$, at the same time as the Dirac $\alpha$ 's have been retained. ${ }^{19}$ The second part of this procedure is only approximately correct since removal of the spin-orbit term will also affect $\alpha$; it is only the spin-orbit term in Eq. (A4) which makes $R_{l}$ distinct from $R_{-l-1}$. This is particularly important for an $\alpha$ component corresponding to a resonant state, e.g., a $d$ state. Slightly above the top of the $d$ band, $\alpha$ has two or one singularities, depending on whether spinorbit coupling is included or not. When spin-orbit coupling $i$ included $\alpha_{d}$ runs continuously from $+\infty$ to $-\infty$ between the singularities (Fig. 16), so $\alpha_{d}$ takes on all the values it had within the resonant $d$ band; $\beta_{d}$, however, has opposite signs outside and between the singularities. Therefore, when we tried to remove spin-orbit coupling by the approximate procedure of neglecting $\beta$ without modifying $\alpha$, a false narrow $d$ band was found slightly above the top of the "true" $d$ band.

In conclusion, the third term of Eq. (A1) should not strictly be called the spin-orbit term, since the second term also contains spin-orbit effects. A pitfall is the spin-orbit mixing form of the third term, but it should be kept in mind that an APW does not have a definite $z$ component of spin; $\left\langle m_{i}\right|$ is the spin component of merely the plane-wave part of the APW.

\section{APPENDIX C: SPIN-ORBIT COUPLING PARAMETER}

When spin-orbit coupling is treated as a perturbation on a bound state of a spherically symmetric potential the spin-orbit coupling parameter is

$$
\begin{aligned}
\frac{1}{2} \xi_{n, l} & =A_{n, l}^{-1} \int_{0}^{\infty}\left(V^{0} / r c^{2}\right) R_{n, l}^{2} r^{2} d r, \\
\text { where } \quad A_{n, l} & \equiv \int_{0}^{\infty} R_{n, l}^{2} r^{2} d r
\end{aligned}
$$

and the spin-orbit term is given by $\mathrm{Eq}$. (1). For the case of a nonbound level of a muffin-tin potential, we shall take the upper limit of the integrals to be the muffin-tin radius $S$, and the quantum number $n$ will be substituted by the positive energy $E$. Since $V^{\prime} / r c^{2}$ is confined to the inner parts of the core, where the atomic and muffin-tin potentials are equal (Fig。15), the spin-orbit parameter of an atomic bound $d$ state and that of the corresponding virtual $d$ state of the muffin-tin potential are approximately equal .

In order to compare with perturbation calculations we shall extract a spin-orbit parameter from the "logarithmic derivatives" $\alpha$ and $\beta$ of a relativistic calculation. When the radial Pauli equation (A4) for $\kappa=-l-1$ is left multiplied by $r R_{l}$ and the equation for $\kappa=l$ is left multiplied by $r R_{-l-1}$ a subtraction of the two resulting equations and a subsequent integration yields

$$
\begin{aligned}
& S^{2} R_{l}(E, S) R_{-l-1}(E, S) \beta_{l}(E) \\
& \quad=(2 l+1) \int_{0}^{S}\left(V^{\prime} / r c^{2}\right) R_{l} R_{-l-1} r^{2} d r .
\end{aligned}
$$

In a similar way, ${ }^{71}$ the relationship between the value of the radial function at the MTS and the normalization integral over the sphere may be found

$$
\left(S R_{\kappa}(E, S)\right)^{2} \frac{\partial}{\partial E}\left(\frac{-R^{\prime}}{R}\right)_{\kappa}=A_{\kappa}(E) .
$$

If we define a spin-orbit coupling parameter as follows:

$$
\begin{aligned}
\frac{1}{2} \xi_{l}(E) \equiv & {\left[A_{l}(E) A_{-l-1}(E)\right]^{-1 / 2} } \\
& \times \int_{0}^{s}\left(V^{\prime} / r c^{2}\right) R_{l} R_{-l-1} r^{2} d r .
\end{aligned}
$$

Equations (C 3 ) and (C4) with $\kappa=-l-1$ and $\kappa=l$ yield the result

$$
\begin{aligned}
& \frac{1}{2} \xi_{l}(E) \\
& \quad=\left|\beta_{l}(E)\right|\left\{(2 l+1)^{2}\left[\frac{\partial}{\partial E}\left(\frac{R^{\prime}}{R}\right)_{l}\right]\left[\frac{\partial}{\partial E}\left(\frac{R^{\prime}}{R}\right)_{-l-1}\right]\right\}^{-1 / 2} \\
& \left.=\left|\beta_{l}(E)\right|\left[\left(\dot{\alpha}_{l}\right)^{2}-\dot{\alpha}_{l} \dot{\beta}_{l}-l(l+1)\left(\dot{\beta}_{l}\right)^{2}\right]^{-1 / 2}, \quad \text { (C } 6\right)
\end{aligned}
$$

where a dot denotes $\partial / \partial E$ 。 Spin-orbit parameters derived from Eq. (C6), but using Dirac $\alpha$ 's and $\beta$ 's, have been shown in Fig. 3, and comparing to Fig. 16 the weak-energy dependence of $\xi$ as opposed to that of $\beta$ may be appreciated.

It may further be shown that, with the knowledge of appropriate spin-orbit parameters, approximately depending on energy, spin-orbit coupling may be added as a perturbation in an APW calculation by using the APW matrix [Eq.(A1)], with $\alpha$ being found from a spin-orbit decoupled radial equation, containing mass-velocity and Darwin terms ad libitum, using Eq. (A2a) and $\beta$ being found from Eq. (C6) which now reads

$$
\beta_{l}(E)=-\frac{1}{2} \xi_{l} \dot{\alpha}_{l}(E) \text {. }
$$

\footnotetext{
${ }^{\dagger}$ Work partially supported by Grant No. GP-7659 from the National Science Foundation.

* Present address: Universitetets Fysiske Laboratorium I., Universitetsparken 5. Copenhagen, Denmark.

${ }^{1}$ See, for instance, M. A. Jensen and K. Andres,
}

Phys. Rev. 165, 545 (1968) and references contained therein.

${ }^{2}$ See, for instance, J. R. Schrieffer, J. Appl. Phys . 39, 642 (1968) and references contained therein.

${ }^{3}$ For these metals there existed one quantitative band- 
structure calculation prior to our work, namely, an APW calculation on Pd by A. J. Freeman, J. O. Dimmock, and A. M. Furdyna [see abstract in J. Appl. Phys. 37, 1256 (1966)]. This calculation was not relativistic, and it concentrated on the gross features of the energy bands, density of states, and Fermi-surface topology. Most recently this calculation has been extended by adding spin-orbit coupling in the tight-binding approximation using the combined interpolation scheme (Ref. 8), and detailed density-of-states histograms have been calculated. Also different potentials have been investigated, and the results obtained with that potential (Hartree-Fock-Slater $4 d^{10}$; Ref. 22), for which the best agreement with experiments were found, are very similar to the results given in the present paper [F. M. Mueller, A. J.Freeman, J. O. Dimmock, and A. M。 Furdyna, Phys. Rev. B 1, 4617 (1970).

${ }^{4}$ The RAPW calculation on Pt by A. R. Mackintosh [Bull. Am. Phys. Soc. 11, 215 (1966)] is the forerunner of the present work, and through the courtesy of Professor Mackintosh many results of his Pt calculation are published here for the first time.

${ }^{5}$ T. L. Loucks, Augmented Plane Wave Method (Benjamin, New York, 1967).

${ }^{6}$ O. K. Andersen and A. R. Mackintosh, Solid State Commun. 6, 285 (1968); O.K. Andersen, thesis, Technical University of Denmark, 1969 (unpublished).

${ }^{7}$ V. Heine, Phys。Rev。153, 673 (1967).

${ }^{8}$ F。 M. Mueller, Phys。 Rev. 153, 659 (1967)。

${ }^{9}$ A. E. Dixon and J. Grodski, Solid State Commun. 7, 735 (1969)

${ }^{10}$ L. R. Windmiller, J. B. Ketterson, and S. Hörnfeldt, J. Appl. Phys. 40, 1291 (1969)。

${ }^{11}$ See, for instance, Ref。26, Fig。 4.

${ }^{12}$ L. F. Mattheiss, Phys。Rev。133, A1399 (1964).

${ }^{13} \mathrm{D}$. Liberman, J.T. Waber, and D。 T. Cromer, Phys. Rev. 137, A27 (1965)。

${ }^{14}$ J. M. Ziman, Proc. Phys. Soc. (London) 91,701 (1967).

${ }^{15}$ L. J. Sham and W. Kohn, Phys. Rev. 145, 561 (1966) and references contained herein. See also Sec. $\mathrm{V}$ of the present paper.

${ }^{16}$ D。 D。 Koelling, Phys. Rev. 188,1049 (1969).

${ }^{17} \mathrm{G}$. A. Burdick, Phys。Rev。 $\underline{129}, 138$ (1963).

${ }^{18} \mathrm{~J}$. Friedel, P. Lenglart, and G. Leman, J. Phys。 Chem. Solids 25, 781 (1964); G。Allan, thesis, University of Paris, 1967 (unpublished); P. Lenglart, Ann. Phys。(N。Y.) 3, 27 (1968)。

${ }^{19}$ L. F. Mattheiss, Phys。Rev. 151, 450 (1966).

${ }^{20}$ The complete data for the constant-energy contours may be obtained from the author.

${ }^{21}$ T. L. Loucks, Phys。Rev. 139, A1333 (1965).

${ }^{22} \mathrm{~F}$. Herman and S. Skillman, Atomic Structure Calculations (Prentice-Hall, Englewood Cliffs, N。J。, 1963).

${ }^{23}$ J. C. Phillips and F. M. Mueller, Phys. Rev. 155, 594 (1967).

${ }^{24}$ D. D. Koelling, A. J. Freeman, and F. M. Mueller Phys. Rev. B 1, 1318 (1970).

${ }^{25}$ L. Hodges, H. Ehrenreich, and N。D。Lang, Phys. Rev. 152, 505 (1966).

${ }^{26}$ J. W. D. Connolly, Phys. Rev。159, 415 (1967).

${ }^{27}$ Near a saddle point in $E(k)$ the constant-energy surfaces are hyperboloids of one and two sheets. For a $P_{1}$ saddle point there is one sheet at energies below the crit- ical energy $E_{s}$, and two sheets above; for a $P_{2}$ saddle point the situation is reversed. In the two-sheet region $N(E)$ contains the van Hove term $\propto\left|E-E_{s}\right|^{1 / 2}$. This holds for the saddle points considered in the present work, unless the saddle point is at symmetry point $L$ and $\Lambda$ is not the axis of the saddle point. For the more complicated cases see J. C. Phillips, Phys. Rev。 104, 1263 (1956).

${ }^{28}$ The calculation actually showed $L 5$ to disappear above the Pd Fermi level, but as this extremely small hole pocket has not been seen in the dHvA experiments, it probably does not exist as part of the Pd Fermi surface.

${ }^{29}$ The hexagonal face contains the twofold axis $Q$, but is not a mirror plane. e.g., the reciprocal-lattice point $\Gamma(1,1,-1) 2 \pi / a$ [Fig。 $9(\mathrm{a})]$ is reflected by the hexagonal face through $L\left(\frac{1}{2}, \frac{1}{2}, \frac{1}{2}\right) 2 \pi / a$ into $\left(\frac{4}{3}, \frac{4}{3},-\frac{2}{3}\right) 2 \pi / a$, which is not a reciprocal-lattice point.

${ }^{30}$ These purely $d$-like saddle points were also identified in the tight-binding calculation of Allan (Ref. 18). However, this author concluded that the saddle points were of no importance for the large peak in the density of states, but rather contributed with small satellite peaks. We believe that this conclusion was caused by noise inherent in the rather special histogram technique applied by Allan.

${ }^{31}$ The small van Hove singularity due to the maximum of the fifth band at $L$ has been smeared out in the $\left[E-E\left(P_{1}\right)\right]$ expansion, since its position $E\left(L_{4}^{+} L_{5}^{+}\right)$was probably not accurately determined in the present calculation (see Ref. 28).

${ }^{32}$ D. W. Budworth, F. E. Hoare, and J. Preston, Proc. Roy. Soc. (London) A257, 250 (1960)。

${ }^{33}$ M. Dixon, F. E. Hoare, and T.M. Holden, Proc。 Phys.Soc。(London) 90, 253 (1967).

${ }^{34} \mathrm{M}$. Dixon et al., in Proceedings of the Confarence on the Electronic Structure of Alloys, Sheffield, 1963 (unpublished)。

${ }^{35}$ B.W. Veal and J.A. Rayne, Phys. Rev。 135, A442 (1964).

${ }^{36} \mathrm{G}$. E. Shoemake and J。A. Rayne, Phys。Letters 26A, 222 (1968)。

${ }^{37}$ W. L. McMillan, Phys. Rev. 167, 331 (1968).

${ }^{38} \mathrm{~F}$. E. Hoare and B. Yates, Proc. Roy。 Soc. (London) A240, 42 (1957).

${ }^{39}$ The width of the peak $\left[E\left(P_{1}\right)-E\left(P_{2}\right)\right]$ is of the order of $10 \mathrm{mRy}$, which for the LXW5 surfaces corresponds to linear $\overrightarrow{\mathrm{k}}$ space dimensions in the order of $\frac{1}{20}$ reciprocal-lattice spacings (in $\langle 100\rangle$ directions)。A $d$-electron mean free path capable of smearing out this peak should therefore be of the order of 20 lattice constants or less, which seems reasonable.

${ }^{40}$ A. Y。-C. Yu and W. E. Spicer, Phys。Rev。 169, 497 (1968).

${ }^{41}$ E. Bucher, W. F. Brinkman, J. P. Maita, and H. J. Williams, Phys. Rev. Letters 18, 1125 (1967).

${ }^{42}$ K. P. Gupta, C. H. Cheng, and P.A. Beck, Phys. Rev. 133, A203 (1964); C. G. Robbins, H. Claus, and P. A. Beck, J. Appl. Phys. 40, 2269 (1969).

${ }^{43}$ A. M. Clogstron, V. Jaccarino, and Y. Yafet, Phys. Rev。134, A650 (1964)。

${ }^{44}$ T. A. Seitchik, A. C. Gossard, and V. Jaccarino, Phys. Rev. 136, A1119 (1964).

${ }^{45}$ S. Foner and E. J. McNiff, Jr., Phys. Rev. Letters $\underline{19}, 1438(1967)$. 
${ }^{46}$ We have used a $g$ factor of 2 for both Pd and Pt. [See further S. Hörnfeldt, J. B. Ketterson, and L. R. Windmiller, Phys. Rev. Letters 23, 1292 (1969); J. Appl. Phys. 41, 1232 (1970); and F. M. Mueller, A. J. Freeman, and D. D. Koelling, ibid. 41, 1229 (1970).]

${ }^{47}$ E. P. Wohlfarth and P. Rhodes, Phil. Mag. 7, 1817 (1962); E. P. Wohlfarth, Phys。Letters $\underline{22}, 280$ (1966)。

${ }^{48} \mathrm{By}$ writing the formula in this way, we wish to emphasize that the assumption of Wohlfarth and Foner of a magnetization- and temperature-independent exchange interaction $\mathrm{V}$, leads to the general expression

$$
B / \sigma=1 / \chi_{0}+f(\sigma, T), \quad f(0,0)=0
$$

where $f$ is independent of exchange interactions and is solely a function of the band density of states $N(E)$. (See also Ref. 50.)

${ }^{49}$ R. Doclo, S. Foner, and A. Narath, J. Appl. Phys. 40, 1206 (1969).

${ }^{50}$ O. K. Andersen, J. Appl. Phys. 41, 1225 (1970)。

${ }^{51}$ S. Foner and E. J. McNiff, Jr., Phys. Letters 29A, 28 (1969).

${ }^{52} \mathrm{G}$. Chouteau, R. Fourneaux, K. Gobrecht, and R. Tournier, Phys. Rev. Letters 20, 193 (1968).

${ }^{53} \mathrm{P}$. Lederer and D. L. Mills, Phys. Rev. 165, 837 (1968); Phys. Rev. Letters 20, 1036 (1968).

${ }^{54}$ P. T. Coleridge, Proc. Roy. Soc.(London) A295, 458 (1966).

${ }^{55} \mathrm{~J}$. B. Ketterson, L. R. Windmiller, and S. Hörnfeldt, Phys。Letters 26A, 115 (1968).

${ }^{56} \delta_{4}$ was obtained from the $L 4$ data

${ }^{57}$ F. M. Mueller, Phys. Rev. 148, 636 (1966); see also L. L. Foldy, ibid. 170, 670 (1968).

${ }^{58}$ F。M. Mueller and M。 G。 Priestley, Phys。 Rev。 148,638 (1966)。

${ }^{59}$ J. J. Vuillemin and M. G. Priestley, Phys. Rev. Letters 14, 307 (1965); J. J. Vuillemin, Phys. Rev. 144, 396 (1966)。
${ }^{60} \mathrm{~J}$. B. Ketterson (private communication).

${ }^{61} \mathrm{~J}$. B. Ketterson and L. R. Windmiller, Phys. Rev. Letters 20。 321 (1968); L. R. Windmiller and J。 B。 Ketterson, ibid. 20, 324 (1968); and (to be published). See also J. B. Ketterson, F. M. Mueller, and L. R. Windmiller, Phys. Rev. 186, 656 (1969)。

${ }^{62} \mathrm{~J}$. B. Ketterson, L. R. Windmiller, S. Hörnfeldt, and F.M. Mueller, Solid State Commun。 6 , 851 (1968).

${ }^{63}$ Some justification for the use of a $\vec{k}$-independent effective exchange and correlation potential of the Slater type have recently been given for the electron gas by L. Hedin, B. Lundquist, and S. Lundquist J. Res. Nat. Bur. Std. (U. S。) (to be published). For an investigation concerning atoms see J. C. Slater, T. M. Wilson, and J. H. Wood, Phys. Rev. 179, 28 (1969); Ref. 65.

${ }^{64}$ For Pd and $\mathrm{Pt}$ the energy shifts induced by including the nonmuffin-tin part of the potential outside MTS was recently calculated (Ref.24), and found not to be very important. The shift in the $L_{6}^{-}-L_{4}^{+} L_{5}^{+} p-d$ separation was 7 and $9 \mathrm{mRy}$ for Pd and Pt, respectively.

${ }^{65} \mathrm{~J}$. C. Slater Mass. Inst. Technol. Solid-State Mol. Theor. Group Semi-Annual Progr. Rept。71, 3 (1969).

${ }^{66} \mathrm{E}$. C. Snow and J. T. Waber, Phys. Rev. 157, 570 (1967)。

${ }^{67}$ The criterion for selecting these state-density calculations was that the width of the histogram boxes should be somewhat less than the width of the peak, i.e., $<5 \mathrm{mRy}$.

${ }^{68} \mathrm{~J}$. O. Dimmock (private communication with T. L. Loucks and L. F. Mattheiss)。

${ }^{69}$ U. Rössler, Solid State Commun. 5, 45 (1967).

${ }^{70}$ D. D. Koelling, Mass. Inst. Technol. Solid-State Mol. Theor. Group Quarterly Progr. Rept. 68 , 36 (1968).

${ }^{71}$ J. Callaway, Energy Band Theory, (Academic, New York, 1964)。 